\title{
Response of Monkey MST Neurons to Optic Flow Stimuli with Shifted Centers of Motion
}

\author{
Charles J. Duffy a and Robert H. Wurtz \\ Laboratory of Sensorimotor Research, National Eye Institute, Bethesda, Maryland 20892
}

\begin{abstract}
Neurons in the dorsal region of the medial superior temporal area (MSTd) have previously been shown to respond to the expanding radial motion that occurs as an observer moves through the environment. In previous experiments, MSTd neurons were tested with radial and circular motion centered in the visual field. However, different directions of observer motion, relative to the direction of gaze, are accompanied by visual motion centered at different locations in the visual field. The present experiments investigated whether neurons that respond to radial and circular motion might respond differently when the center of motion was shifted to different regions of the visual field. About $90 \%$ of the $\mathbf{2 4 5}$ neurons studied responded differently when the center of motion was shifted away from the center of the field. The centers of motion preferred by each neuron were limited to one area of the visual field. All parts of the visual field were represented in the sample, with greater numbers of neurons preferring centers of motion closer to the center of the field. We hypothesize that each of the MSTd neurons has a center of motion field with a gradient of preferred centers of motion, and that there is an orderly arrangement of MSTd neurons with each region of the visual field being represented by a set of neurons. This arrangement creates the potential for graded responses from individual neurons for different directions of heading as an observer moves through the environment.
\end{abstract}

[Key words: visual system, motion, optic flow, extrastriate cortex, MST, monkey, FOE]

While moving through the environment, the visual world streams around observers in a pattern which reflects their motion. These optic flow fields combine the effects of all observer movements in three-dimensional space to provide visual information that can guide self-motion, stabilize posture, and reveal the structure of the environment (Gibson, 1950, 1986). All observer movements consist of translation along, and/or rotation around, three axes in space, and similarly all optic flow fields consist of visual patterns created by these motion components.

Attempts to investigate the neuronal processing underlying the analysis of optic flow fields have concentrated on the medial superior temporal area (MST) of the macaque superior temporal

\footnotetext{
Received Nug. 2, 1994; revised Feb. 23, 1995; accepted Feb. 27, 1995.

Correspondence should be addressed to Robert H. Wurtz, Laboratory of Sensorimotor Research, National Eye Institute, Building 49, Room 2A50, Bethesda, MD 20892-4435.

Present address: Departments of Neurology, Neurobiology and Anatomy, and Ophthalmology, and the Center for Visual Science, University of Rochester Medical Center, Rochester, NY 14642.

Copyright (C) 1995 Society for Neuroscience $0270-6474 / 95 / 155192-17 \$ 05.00 / 0$
}

sulcus. Neurons in this region show direction selective visual motion responses (Desimone and Ungerleider, 1986; Tanaka et al., 1986; Ungerleider and Desimone, 1986). Those in dorsal MST (area MSTd) have very large receptive fields, often encompassing more than a quadrant of the visual field, and are most responsive to large pattern motion (Tanaka et al., 1986; Komatsu and Wurtz, 1988). Studies in anesthetized monkeys showed that these neurons respond selectively to expanding or rotating patterns (Saito et al., 1986; Tanaka et al., 1986, 1989; Tanaka and Saito, 1989; Saito, 1993) which possess some of the characteristics of optic flow fields. Studies in awake monkeys (Andersen et al., 1990, 1993; Wurtz et al., 1990; Duffy and Wurtz, 1991a,b; Orban et al., 1992; Graziano et al., 1994; Lagae et al., 1994) have demonstrated that many of these neurons respond selectively to many of the elements of optic flow, including radially expanding stimuli.

The response to radially expanding stimuli is of particular interest because it is seen by observers as they move forward. In a previous study of MSTd neurons (Duffy and Wurtz, 1991), we used computer-generated patterns of optic flow in which the radial or circular center of motion was usually in the center of the monkey's visual field. One possibility is that these neurons might respond better if that center of motion were shifted to different regions of the visual field. To test this possibility, we studied MSTd neurons with large field optic flow stimuli using centers of motion shifted to different regions of the visual field. We find that the response of many MSTd neurons is altered when the center of motion is shifted, and that there are neurons in MSTd that respond best to such shifted centers of motion. We hypothesize that these neurons form a neuronal map of centers of motion, and that such a map might contribute to the determination of heading based on optic flow.

Brief reports of this work have appeared previously (Duffy and Wurtz, 1991c, 1993; Wurtz and Duffy, 1992; Wurtz et al., 1993).

\section{Materials and Methods}

Behavioral and physiological procedures. Two adult Rhesus monkeys (Macaca mulatta, subjects $79 \mathrm{~N}$ and $26 \mathrm{~K}$ ) were trained to sit in a primate chair and perform a visual fixation task for liquid reward. The monkeys sat in the chair for several hours per day and then were returned to their home cages. All experimental protocols were approved by the Institute Animal Care and Use Committee and complied with Public Health Service Policy on the humane care and use of laboratory animals.

The monkeys were prepared for recording in a single surgical session using procedures described previously (Duffy and Wurtz, 1991a). After anesthetic induction (ketamine $10 \mathrm{mg} / \mathrm{kg}$ and atropine $0.04 \mathrm{mg} / \mathrm{kg}, \mathrm{i} . \mathrm{m}$.) and endotracheal intubation, inhaled Isoflurane was used to maintain a surgical plane of general anesthesia. Scleral search coils were implanted bilaterally (Judgc et al., 1980), recording cylinders were placed over parietal cortex bilaterally (AP -2, ML 15), and a stainless steel head 
holder was embedded in a dental acrylic cap that covered the top of the skull. Postoperative analgesia (Flunixin, $2 \mathrm{mg} / \mathrm{kg}$, i.m.) was administered as judged appropriate by the attending veterinarian.

After recovering from surgery, the monkeys were trained to fixate a spot of light on the tangent screen in front of them; eye position was monitored by the magnetic search coil technique (Robinson, 1963). The monkey sat $50 \mathrm{~cm}$ away from the center of a $100^{\circ} \times 100^{\circ}$ tangent screen. Each trial began with the appearance of the projected image of a red light-emitting diode (LED: $0.25^{\circ}$ in diameter, $2.7 \mathrm{~cd} / \mathrm{m}^{2}$ ) at the center of the screen. The monkey's task was to fixate the LED image within $500 \mathrm{msec}$ and maintain binocular fixation $\left( \pm 2.5^{\circ}\right)$ for $6-7.5 \mathrm{sec}$. At the end of successful trials, a reinforcing tone was sounded and the monkey received a liquid reward on a variable ratio reinforcement schedule. During each fixation period, three stimuli were projected onto the screen in succession. Each stimulus had a duration of $1 \mathrm{sec}$, and interstimulus intervals were between 1 and $1.5 \mathrm{sec}$. Task parameters were adjusted to maintain a high success rate during training, with the monkeys successfully completing $90 \%$ of the experimental trials after a few weeks.

The behavioral task, stimulus timing, and digitizing of single neuron recordings were controlled by the real-time experimental system (REX) (Hays et al., 1982) running on a dedicated PDP 11/73 computer. Single neuron activity was digitized using a window discriminator and sampled at $1 \mathrm{kHz}$ for storage with markers of stimulus and behavioral events. An on-line raster display showed the occurrence of single neuron discharges with markers added to give the temporal relation to stimulus and behavioral events.

Displays used for later data analysis were spike density histograms created by replacing the pulses representing spikes with Gaussian pulses with a width corresponding to a standard deviation of $20 \mathrm{msec}$ (MacPherson and Aldridge, 1979) thereby providing a continuous function. Eye position was monitored by REX for behavioral control during all experiments but was not stored.

Recordings were made in both hemispheres of the two monkeys (79N and $26 \mathrm{~K}$ ). A grid system was placed within the recording cylinders (Crist et al., 1988) to facilitate the insertion of stainless steel guide tubes through the dura to a depth about $5 \mathrm{~mm}$ above the superior temporal sulcus. At the beginning of each recording session, a guide tube stylet was removed and an epoxy coated tungsten microelectrode (Frederick Haer, $1.5-2.0 \mathrm{M} \Omega$ at $1 \mathrm{kHz}$ ) was inserted. The electrode was advanced using a hydraulic microdrive (Narishige) while neuronal activity was monitored to establish the relative depth of landmarks, including gray and white matter layers and neuronal response properties. Studies were initiated only on clearly isolated neuronal discharges in order to increase the probability of stable recording for the several hours required to conduct these experiments.

When the experiments were completed, several electrolytic marks were made along previously recorded penetration tracks in each of three guide tubes. Several days later the monkey was deeply anesthetized with sodium pentobarbital followed by intracardiac perfusion, first with saline then with $10 \%$ formalin. The posterior half of each hemisphere was frozen and cut into $50 \mu \mathrm{m}$ serial sections in the sagittal plane. Every fourth section was stained for cell bodies with thionin, every fifth section was stained for myelineated fibers with a modified silver stain (Gallyas, 1979), and every section was stained through the region of the electrolytic marks. Drawings were made of the relevant sections to establish the locations of the electrolytic marks relative to anatomic landmarks in the superior temporal sulcus. The location of recording sites was extrapolated from the relative position of the guide tubes and the distribution of marks and electrode tracks. We found that roughly $90 \%$ of the neurons were within the densely myelineated zone on the anterior bank of the sulcus that is included in the dorsal-medial region of MST (MSTd) (Komatsu and Wurtz, 1988). The remaining neurons were farther down the anterior bank, but had the same physiological characteristics, and we will consider them separately in this study.

Visual stimuli. When a neuron was isolated, we used hand-held projectors to define its approximate receptive field boundaries to flashed and moving, single spot and random dot pattern, images. This allowed us to determine whether its receptive field characteristics met the criteria of an MSTd neuron: directionally selective motion sensitivity, a large receptive field that was near or included the fovea, and a better response to a large pattern of random dots than to single spots (Komatsu and Wurtz, 1988).

During a trial, the computer running REX triggered the presentation of a pseudorandom sequence of $1 \mathrm{sec}$ visual stimuli which were back projected onto a translucent tangent screen by a TV projector (Sony 900 ). The visual stimuli filled the central $100^{\circ} \times 100^{\circ}$ of the monkey's visual field, and consisted of random dot patterns which were generated off line, stored, and displayed using an 80386 based computer. Each pattern contained 360 dots, each dot subtending $0.75^{\circ}$ at $1.8 \mathrm{~cd} / \mathrm{m}^{2}$ against a $0.2 \mathrm{~cd} / \mathrm{m}^{2}$ background.

All neurons were first studied with a set of 12 stimuli that are components of optic flow stimuli: eight directions of lamellar or planar motion on the tangent screen (with directions at $45^{\circ}$ intervals around $360^{\circ}$ ) which would be produced by various translations of the observer in the frontoparallel plane; two directions of radial motion (inward and outward with the center of motion at the fixation point) which would be produced by forward or backward translation of the observer, two directions of rotations or circular motion (clockwise and counterclockwise with the center of motion at the fixation point) which would be produced by the roll of the observer. The two remaining rotational motions (pitch and yaw) were not used because in our previous experiments (Duffy and Wurtz, 1991a) we found that the response to these stimuli was the same as to the planar stimuli having that direction of motion on the screen; this would be expected given that all the stimulus dots are in one plane. We also used trials with only a blank screen as control trials to determine background activity as well as trials with stationary dot stimuli.

All stimuli contained 360 randomly distributed white dots which were moving in the specified pattern as soon as they appeared on the screen. All movement algorithms included dot wrapping and limited dot halflives to maintain a constant and uniform dot density across the screen. The average dot speed was $40^{\circ} / \mathrm{sec}$ in all stimuli, a speed at which MST neurons consistently respond (Tanaka and Saito, 1989; Duffy and Wurtz, 1991a). In planar stimuli, all dots moved at that speed. In radial and circular stimuli, dot speed varied in proportion to a dot's distance from the center of motion in the pattern. In these stimuli the average dot speed was $40 \% \mathrm{sec}$, which is comparable to the radial pattern created by forward observer movement of about $45 \mathrm{~cm} / \mathrm{sec}$. The algorithms for generating these stimuli were described previously (Duffy and Wurtz, 1991a). All stimuli appeared as if moving in one plane in front of the monkey simulating motion in a rigid world without independently moving objects. Naturalistic cues, such as changes in the blur, brightness, disparity, size, and texture of moving elements in the display, were not included in these stimuli.

One difference between the stimuli in the present study and in our previous study (Duffy and Wurtz, 1991a) is the positioning of our large field stimuli. In our previous study we positioned the stimuli on the receptive fields of the ncurons, as have others, whereas in the present experiment we always placed the stimuli in the center of the screen (center of gaze) regardless of the location of the receptive field. Our previous experience revealed that the receptive fields of these MSTd neurons are large and come close to the fovea; with our $100^{\circ} \times 100^{\circ}$ stimuli, we found that the precise position in the receptive field made little difference in the results (Duffy and Wurtz, 1991b). In these experiments the fixation point was at the center of the screen because our goal was to test the effect of the same stimuli on all cells rather than to determine receptive field organization.

Radial and circular stimuli with shifted centers of motion were produced by frame-wise vector addition of planar motion to the radial or circular motion in the display. This produced a radial or circular display in which the center of motion was shifted away from the center of the stimulus (and center of the screen). It should be noted that this did not produce the curved dot paths of visual motion that would be generated by point-by-point vector addition of the planar and radial or circular motion in which each dot carries a new motion vector in each frame. Such stimuli simulate the addition of observer rotation (or eye rotation) around the vertical axis with forward translation. In contrast, our shifted center of motion stimuli more precisely simulated forward translation in a direction other than the direction of visual gaze.

We used stimulus sets which combined eight directions of planar motion with inward or outward radial motion and clockwise and counterclockwise circular motion. Figure 1 shows examples for two directions of planar motion (leftward and rightward motion) for each of the radial and circular stimuli. The eight patterns within the frame represent the results of combining the leftward or rightward planar motion (above the frame) with the radial or circular motion (along the left side). The basic radial and circular patterns contain centers of motion which are centered in the stimulus, and the combination stimuli have shifted centers of motion. For example, combining leftward planar motion and 


\section{PLANAR MOTION}

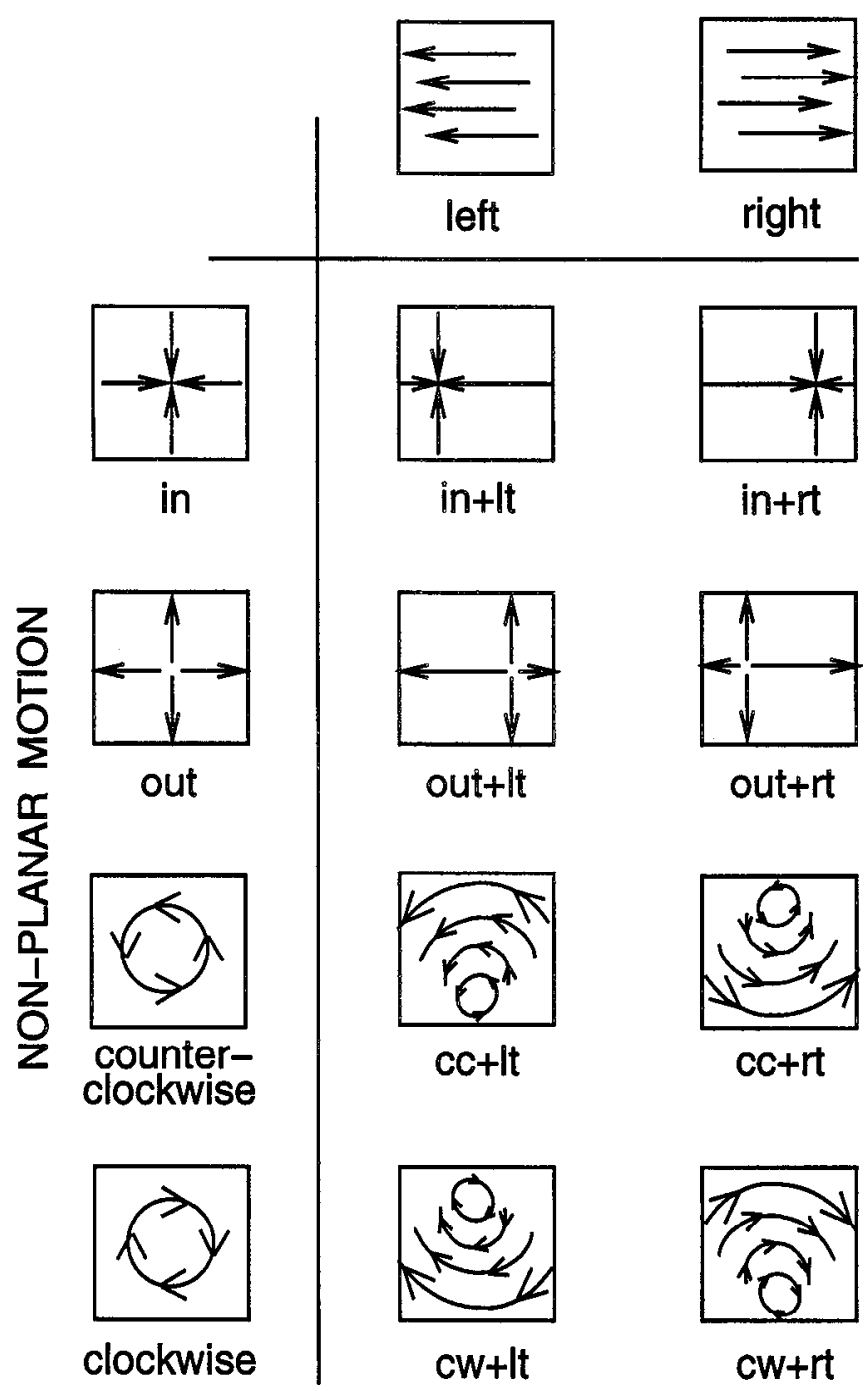

Figure 1. Radial and circular stimuli with shifted centers of motion resulting from the vector addition of planar and radial motion (top two rows), or planar and circular motion (bottom two rows). Each box represents the $100^{\circ} \times 100^{\circ}$ stimulus, and the arrows represent the direction of dot motion. The left column shows the results of combining radial and circular motion with leftward planar motion, and the right column shows the results of such a combination with rightward planar motion. The resulting stimuli with shifted centers of motion contain centers of motion that are shifted $45^{\circ}$ off the center of the stimulus, and the direction of shift reflects the direction of the combined planar motion. The monkey always fixated in the center of the screen, not the center of the stimulus.

inward radial motion produced a radial pattern with the center of motion shifted to the left. Note that not only is the location of the center of motion shifted, but motion throughout the whole flow field is altered by the addition of planar motion to the radial or circular motion.

Analysis of neuronal activity. Neuronal activity was sampled in the $600 \mathrm{msec}$ period beginning $400 \mathrm{msec}$ after stimulus onset. We ignored the first $400 \mathrm{msec}$ after stimulus onset because in our previous study (Duffy and Wurtz, 1991a) we found that the activity in this early response period was less well related to the specific stimulus than was the response in the later period. We compared the response in this 600 msec period to the activity in a 600 msec period on a trial when the monkey fixated but no stimulus was presented. We tested all responses for statistical significance using a Student's $t$ test (significance level of 0.01 ), comparing activity in 6-7 stimulus presentations to activity dur ing an equal number of control trials.
We also measured the strength of the relationship between the location of the center of motion and the neuronal response. We plotted the strength of the response to each shifted center of motion stimulus on a polar plot, with the direction of the center of motion as the angle and the response amplitude as the radius. We used circular statistics (Batschelet, 1981) to measure the strength of the relationship between the direction in which the center of motion is shifted and the amplitude of the neuronal response. We tested whether the distribution of response amplitudes around $360^{\circ}$ deviated significantly from randomness by deriving the Rayleigh $z$ statistic for each distribution using a significance level of $p<0.01$. The significance of the $z$ values can be interpreted as indicating a clear location preference for shifted centers of motion.

\section{Results}

\section{Responses to planar, radial, and circular motion}

We studied the responses of 268 neurons on the anterior bank of the superior temporal sulcus. All neurons had large receptive fields that frequently included the fovea and a full quadrant of the visual field, and these neurons responded better to the motion of large patterned stimuli than to the motion of single spots. These characteristics are typical of neurons in the dorsal region of this MST area, MSTd (Komatsu and Wurtz, 1988).

For each cell, we first determined the responses to a standard set of 12 components of optic flow stimuli (shown at the top of Fig. 2) that were centered on the monkey's fixation point. The motion was planar (eight directions), radial (inward and outward), or circular (counterclockwise and clockwise). As we reported previously (Duffy and Wurtz, 1991a), some neurons responded best only to a single type of visual motion stimulus, either planar, circular, or radial motion, and Figure $2 A$ shows an example of such a single component neuron which responded to outward radial motion (darkened spike density plot). Other neurons responded to two of these motion stimuli such as the double component neuron shown in Figure $2 B$. Others responded to planar, circular, and radial visual motion stimuli, and Figure $2 C$ shows the responses of such a triple component neuron.

Instead of discrete subpopulations of single, double, or triplecomponent neurons, we again found a continuum of response selectivity for the 12 motion stimuli (Duffy and Wurtz, 1991). However, in order to describe the distribution of cells, we classified each neuron according to the types of stimuli that evoked significant responses (Fig. 3). Triple component neurons formed the largest group, most being planocirculoradial (PCR) neurons with selective responses to planar, circular, and radial stimuli. Also included in this group were nonselective excitatory (NSE) and nonselective inhibitory (NSI) neurons which responded to all three types of stimuli (frequently to all 12 stimuli used). Double-component neurons were mainly planoradial (PR) neurons with responses to a planar or radial stimulus and planocircular (PC) neurons with responses to a planar or circular stimulus. We also found a few circuloradial (CR) neurons with responses to a circular or radial stimulus, a category first reported by Graziano et al. (1990), but they are rare in our sample. Single component neurons are of three varieties: planar $(\mathrm{P})$ neurons responded only to planar stimuli, radial (R) neurons responded only to radial stimuli, and circular (C) neurons responded only to circular stimuli.

\section{Responses to shifted centers of motion}

We used stimulus sets with shifted centers of motion which were produced by combining radial motion and circular motion with eight directions of planar motion as illustrated in Figure 1. In our sample of 268 neurons, 245 had significant responses to radial or circular stimuli; they were triple, double, and single 

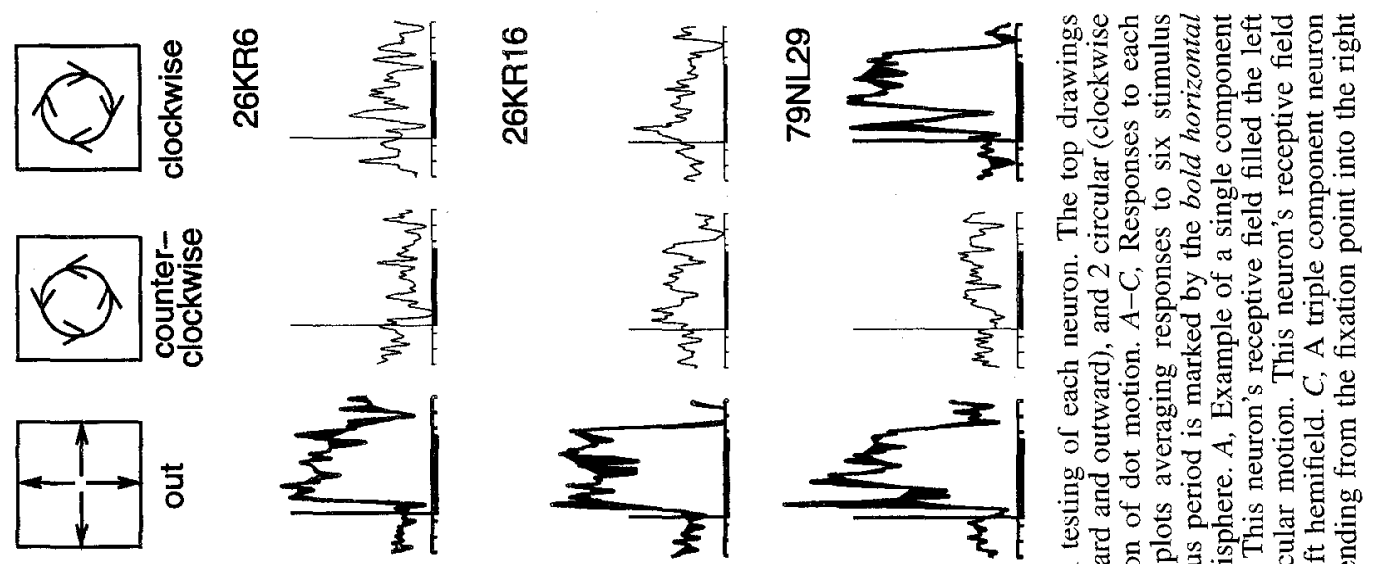

0 宩 980

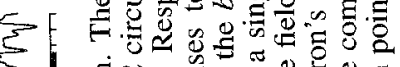

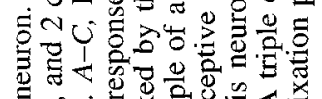

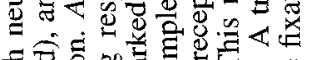
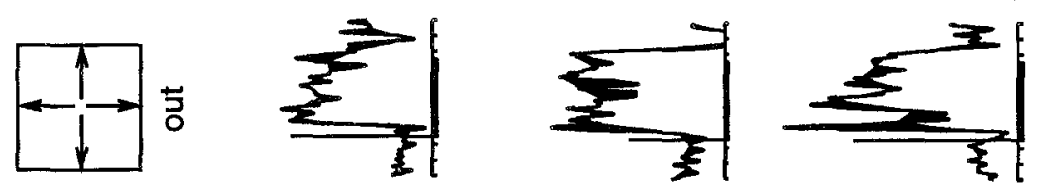

is

o 运

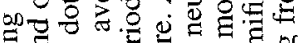

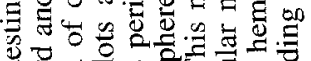

\%
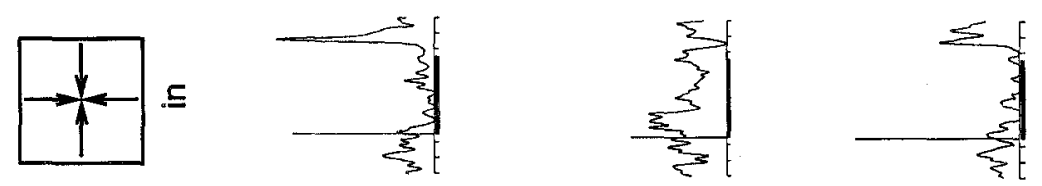

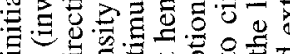

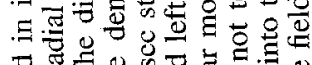

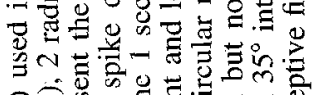

$1 / 1 \pi^{\frac{5}{5}}$
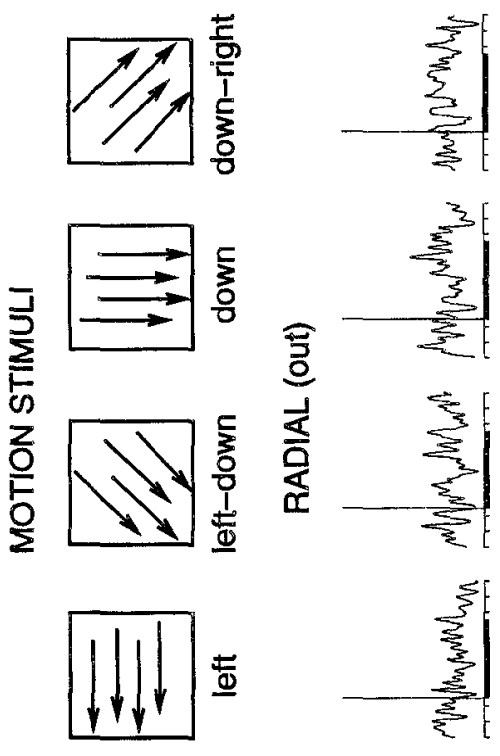

结
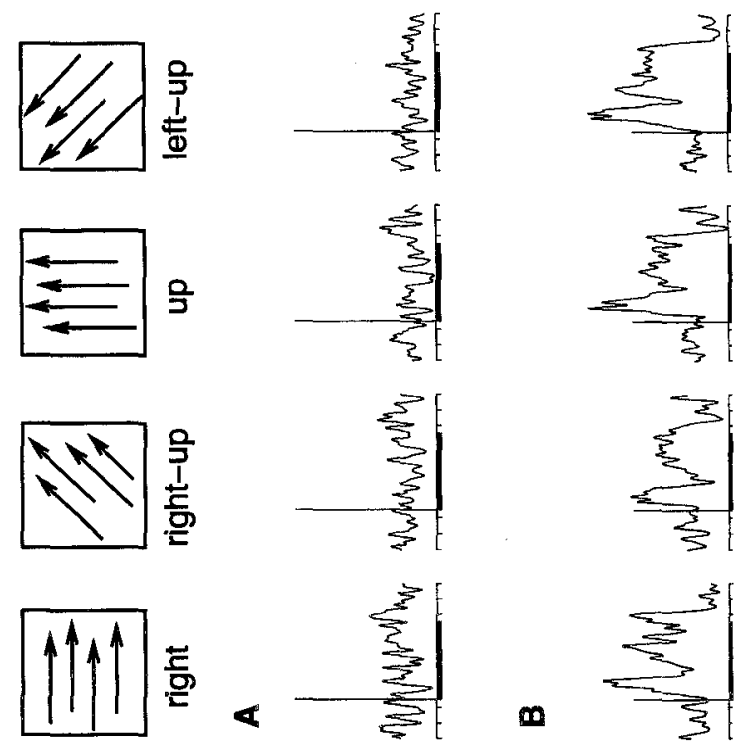
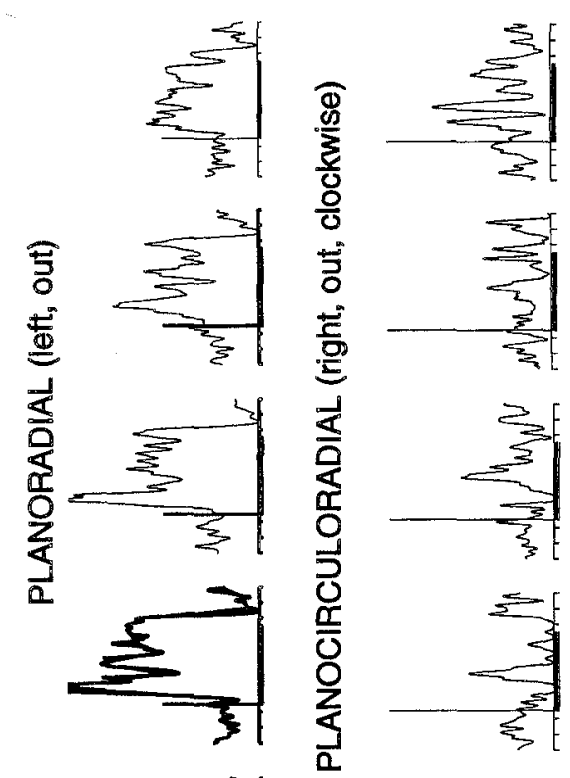

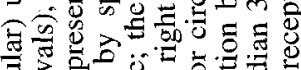

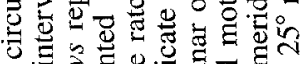

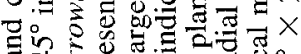

讨 50

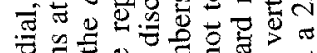

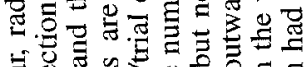

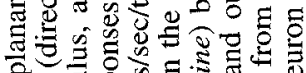

उ.

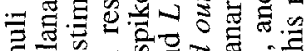

Z F

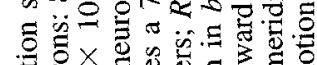

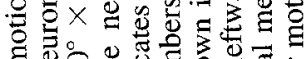

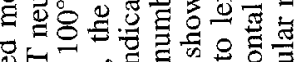

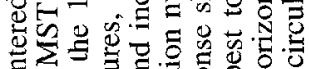

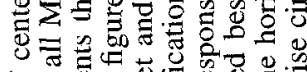

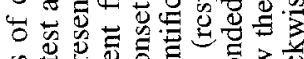

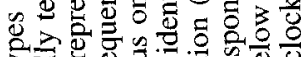

उ.

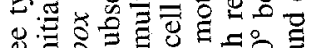

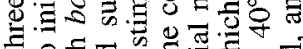

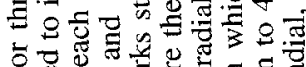

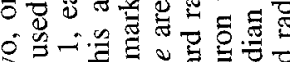

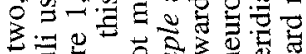

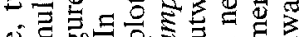

द

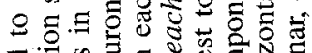

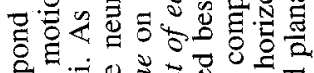

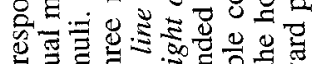

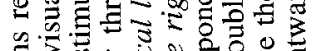

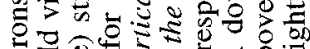

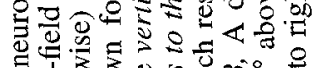

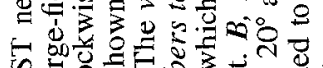

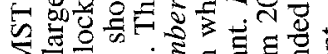

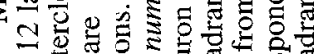
तi

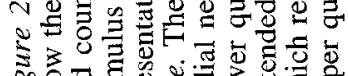




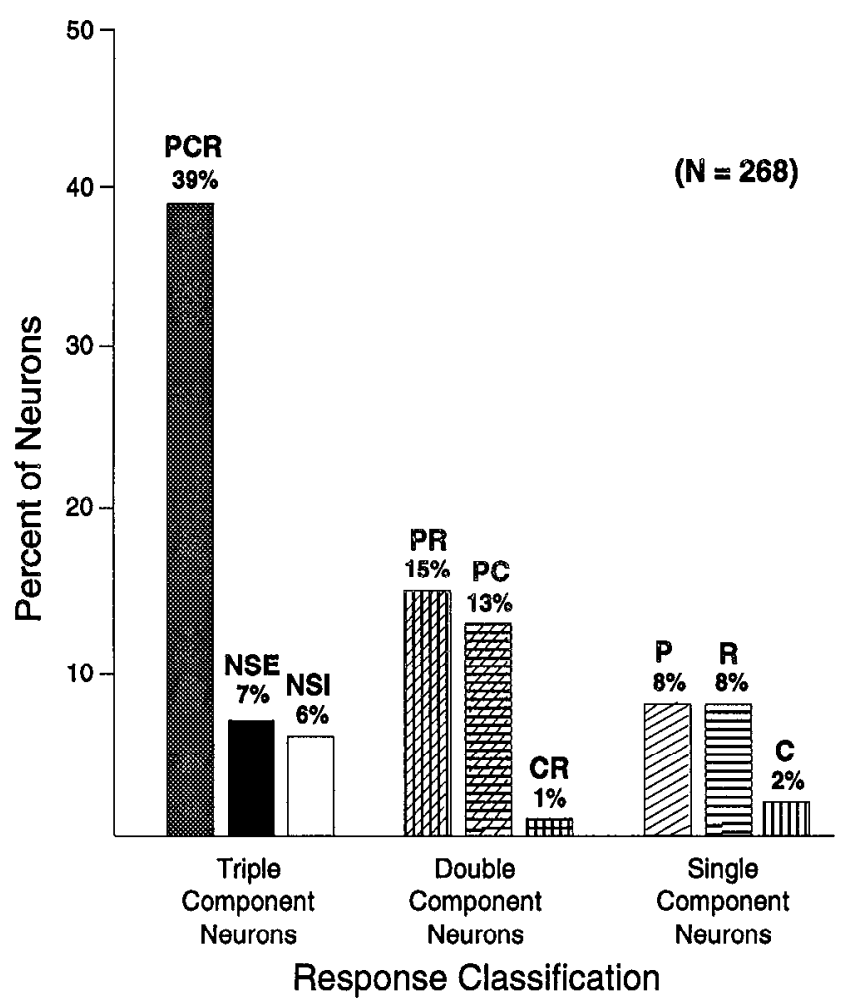

Figure 3. Percentage of neurons responding to one, two, or three types of visual motion stimuli. To be classed as responding to a stimulus, the neuron had a significant response (see Materials and Methods) to at least one of the planar, circular, or radial stimuli shown in Figure 2; $1 \%$ of the neurons failed to meet this criteria and are not shown on the graph. Triple component neurons are: planocirculoradial $(P C R)$, nonselective excitatory (NSE), and nonselective inhibitory $(N S I)$. Double component neurons are: planoradial $(P R)$, planocircular $(P C)$, and circuloradial $(C R)$. Single component neurons are: planar $(P)$, radial $(R)$, circular $(C)$. The majority of neurons $(81 \%)$ respond to more than one type of motion.

component neurons. We tested each of these neurons with either the radial or circular stimuli with shifted centers of motion depending upon whether the radial or circular stimulus evoked the strongest response in the initial study of the neuron. In all cases the monkey continued to fixate at the center of the screen. For the largest group of neurons (43\%), the response remained largest to stimuli with the centers of motion in the center of the screen, and Figure $4 A$ illustrates the responses of one such neuron. The second most common group of neurons $(26 \%)$ had responses that were significantly larger to stimuli with shifted centers of motion (Fig. $4 B$ ). An additional $22 \%$ of the neurons tested had mixed responses to the shift of the center of motion, some smaller, others larger. The remaining $9 \%$ did not respond differently to the stimuli with shifted centers of motion.

Figure $4 C$ shows how frequently the 245 neurons showed significant changes in response amplitude with the shifted center of motion stimuli. About $90 \%$ of the neurons (dark bars) had responses to one or more shifted center of motion stimuli that were significantly different from the responses to the centered stimulus. Thus, a large fraction of the neurons gave a different response to stimuli with shifted centers of motion than they did to the centered stimulus. This suggests substantial response selectivity for the location of the center of motion in these visual motion patterns.

Many neurons showed a systematic preference for centers of motion in only one region of the visual field. This can be illustrated using a polar plot to show the strength of the response for centers of motion shifted in different directions. Figure $5 B$ shows the responses of a neuron with centers of motion primarily in the upper right quadrant. In contrast, the neuron in Figure $5 \mathrm{~A}$ shows no such preference. The strength of this preference for shifts in given directions can be described using the Rayleigh $z$ statistic (see Materials and Methods) for each of the neurons studied with shifted centers of motion. Figure $5 C$ shows the range of $z$ values for our sample of cells, and shows that $65 \%$ of the neurons yielded significant $z$ values (dark bars). These significant values indicate a clear location preference for the direction of shifted centers of motion.

We have so far only considered the effect of shifting the center of motion of the radial or circular stimulus (the preferred stimulus) that gave the best response for each neuron, and we can not reject the possibility that any stimulus with a shifted center of motion would lead to a different response in these cells. We therefore compared responses to stimuli with shifted centers of motion derived from the preferred radial or circular stimuli to those derived from nonpreferred radial or circular stimuli. For example, the neuron in Figure $6 A$ responded strongly to outward radial motion but not to clockwise circular motion, and it responded more strongly to the radial stimuli with shifted centers of motion. The neuron in Figure $6 B$ responded to outward radial motion and only somewhat less to inward radial motion, but still responded most vigorously to the shifted center of motion stimuli derived from outward radial motion. We made such comparisons for 59 neurons, and Figure $6 \mathrm{C}$ shows that most of the neurons $(56 \%)$ showed significantly stronger responses to the preferred stimulus rather than to a nonpreferred stimulus with shifted centers of motion (solid bar). Only $10 \%$ showed stronger responses to the nonpreferred stimuli (open bar) although $34 \%$ of the neurons showed comparable responses to the preferred and nonpreferred stimuli (shaded bar). Five times as many neurons showed an increase in response when the stimuli with shifted centers of motion were derived from the preferred radial or circular stimulus as when they were not. Thus, shifting the center of motion of any radial or circular stimulus did not necessarily improve the response.

One possibility was that the center of motion selectivity revealed by these stimuli was somehow a simple product of some other response characteristic previously described. Two of the most obvious characteristics are the receptive field sizes of the neurons and their responses to the components of optic flow. We had only a sufficient number of experiments on the radial stimuli with shifted centers of motion to address these issues. We first determined center of motion preference and then compared that preference to that neuron's receptive field type, dividing reccptive fields according to their overall extent as determined with a hand-held moving pattern stimuli. Three types of receptive field were recognized: those with sides of about $25^{\circ}\left(15-40^{\circ}\right)$ and restricted to one quadrant of the visual field, those with sides of about $50^{\circ}\left(40-70^{\circ}\right)$ and filling one quadrant, and those with sides of about $75^{\circ}\left(>70^{\circ}\right)$ and filling substantially more than a quadrant. Figure $7 A$ shows the distribution of center of motion preferences for neurons having these different types of receptive fields. All receptive field types showed less preference for planar stimuli, and comparable preference for shifted and radial stimuli, except the largest fields showing fewer preferring radial stimuli. None of these receptive field types was uniquely related to a stimulus preference. 
COMBINATION STIMULI
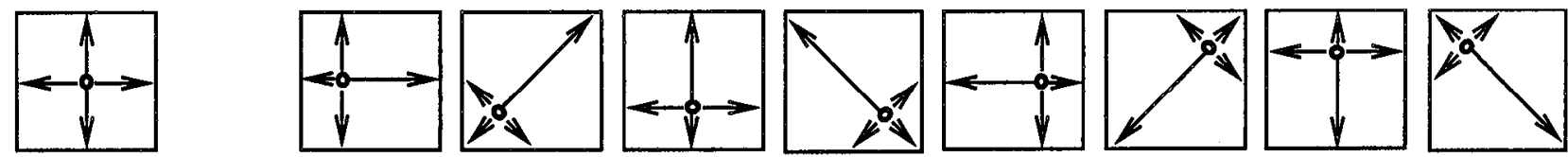

A
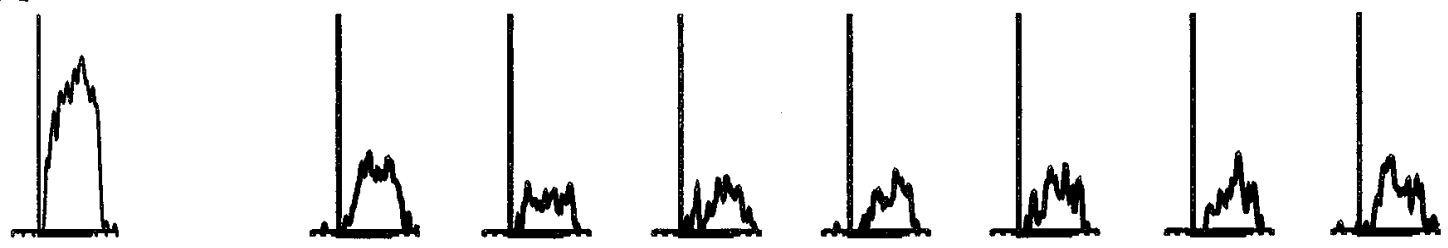

79NL63

B
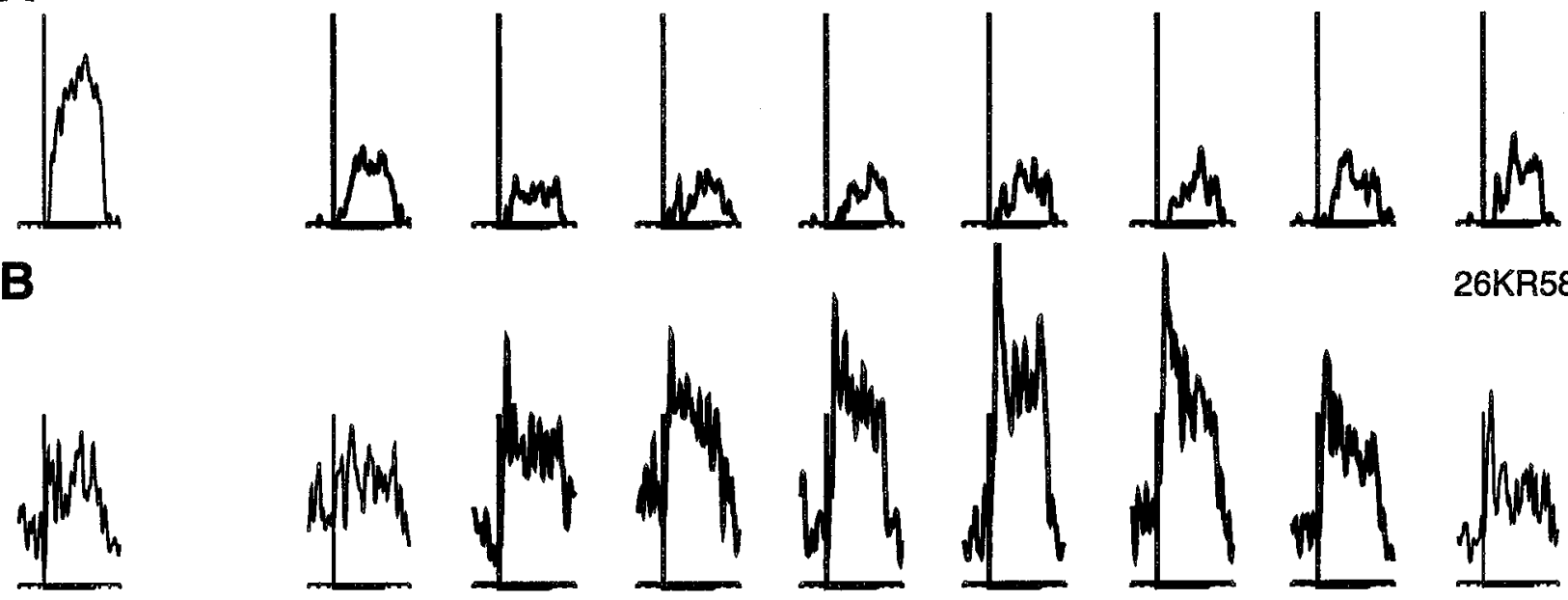

26 KR58

C

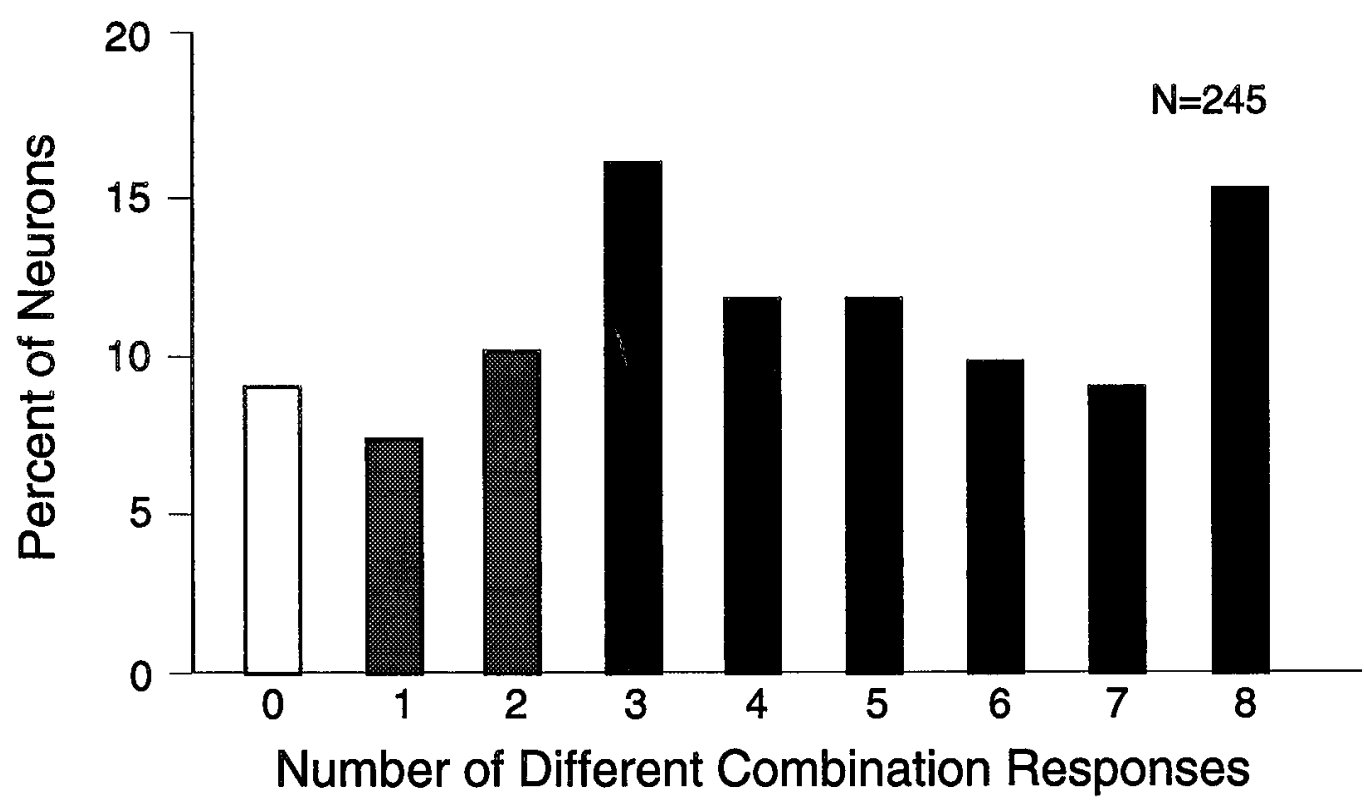

Figure 4. Neuronal responses to stimuli with shifted centers of motion. At the top, drawings show the centered outward radial stimulus (left) and the eight outward radial and planar stimuli with shifted centers of motion (right). $A$ and $B$, Spike density histograms showing the responses of two neurons to the outward radial stimuli and stimuli with shifted centers of motion. Vertical line indicates 75 spikes/sec discharge rate; horizontal bar indicate $1 \mathrm{sec}$ stimulus duration. Responses to stimuli with shifted centers of motion that are significantly different from the centered response $(t$ test, $p<0.01)$ are drawn in bold outline. A, A neuron that responded best when the center of motion was in the center of the stimulus. This neuron is part of a subgroup (14\% of the neurons) that showed significantly smaller responses to all eight of the stimuli with shifted centers. This planocirculoradial neuron's receptive field was $40^{\circ} \times 40^{\circ}$, extending from the fixation point into the right lower quadrant. $B$, A neuron that was activated by any stimulus containing outward motion although the strongest responses were evoked by stimuli with centers of motion near the right edge. This planocirculoradial neuron's receptive field included all of the $50^{\circ} \times 50^{\circ}$ area of the left lower quadrant, except the $5^{\circ}$ closest to the vertical meridian. $C$, Frequency with which neurons responded to shifted center of motion stimuli. Each bin in the histogram shows the percentage of neurons with significant differences between the responses to the indicated number of shifted center of motion stimuli and the centered motion stimulus ( $t$ test, $p<0.01$ ). Over $90 \%$ of the ncurons showed a change (dark bars).

We next examined whether we could predict the response of a neuron to the stimuli with shifted centers of motion if we knew the neuron's response to each of the planar, radial, and circular stimuli (the single, double, or triple component response categories shown in Fig. 2). We suspected that neurons responding to several optic flow components (nonselective excitatory, planocirculoradial, and planoradial) would be the ones most responsive to the stimuli with shifted centers of motion; we thought that they might be tuned to shifted centers and that our categorization of them was simply the result of inadequate stimuli 

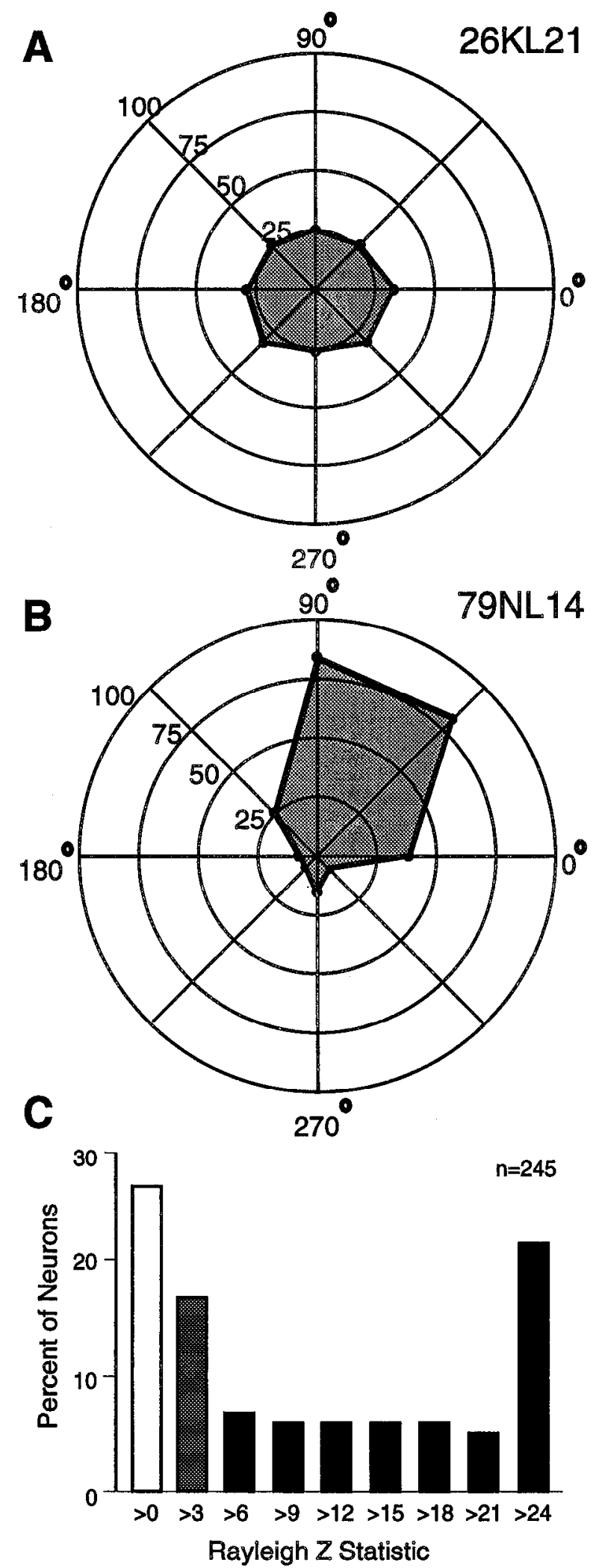

Figure 5. Strength of directional preference for shifted centers of motion. $A$ and $B$, Polar plots showing response amplitude (radius in spikes/sec) versus direction in which the center of motion was shifted (angle in degrees). A, Polar plot of a neuron showing no clear directional preference, with similar response amplitudes for all eight directions in which the center of motion was shifted. $B$, The neuron shows strong directional preference and responded most vigorously when the center of motion was shifted upwards $\left(90^{\circ}\right)$. This radial neuron's receptive field in our previous experiments. This turned out not to be the case. In Figure $7 B$, the percentage of neurons that responded best to the stimuli with shifted centers of motion was largely the same across all the categories in which the neurons responded to other than just planar stimuli. In contrast, neurons that responded only to the planar motion in the set of 12 optic flow component stimuli, showed the highest percentage of neurons $(69 \%)$ that responded best to the shifted center of motion stimuli. We conclude that the shifted center of motion stimuli reveal an aspect of neuronal response selectivity that is not predicted by significant responses to the components of the optic flow stimuli.

\section{Spatial distribution of preferred centers of motion}

Because many of the MSTd neurons gave their strongest response to the stimuli in which the center of motion was shifted away from the center of the stimulus, we investigated whether there was any preference among the neurons for one part of the visual field such as above or below the horizon or near or far from the center. Figure $8 \mathrm{~A}$ shows the spatial distribution of the centers of motion of the stimuli we used, with each of the 17 boxes representing the $100^{\circ} \times 100^{\circ}$ stimulus, the arrows representing the direction of dot motion, and the circles representing the center of motion. The stimuli are ordered in Figure $8 \mathrm{~A}$ so that the one with centered motion is in the middle, those with a shifted centers of motion are placed eccentrically in the direction of the shift, and those with planar motion are at the edge of the figure as if their centers of motion were in the far periphery.

We observed all three possible response patterns among the neurons studied with these stimuli: those with their strongest responses to centered, eccentric, or peripheral centers of motion. Figure $8 B-D$ show the responses of three neurons all of which responded to outward radial motion. The spike density histograms of their responses are located in the positions occupied by the stimuli in Figure $8 \mathrm{~A}$. The best responses are darkened, as are the responses that were not significantly different from the best responses. Figure $8 B$ shows the best response to the centered motion with all other responses being significantly smaller. In Figure $8 C$ the best responses are to an eccentric center of motion stimulus, and in Figure $8 D$ the best response is to a planar stimulus, one that can be regarded as having a peripheral center of motion.

Figure $9 A$ shows the distribution of preferred centers of motion for our sample of neurons that responded to outward or inward radial motion and were tested with radial stimuli with shifted centers of motion. We did similar experiments using clockwise and counterclockwise circular motion, and Figure $9 C$ shows the comparable distribution for neurons which preferred these stimuli. The distribution of preferred centers of motion included all of the 17 shifted center sites tested, suggesting that

\section{$\leftarrow$}

included all of the right hemifield. $C$, The distribution of Rayleigh $z$ statistics describing the strength of the directional preferences of the neurons studied with shifted centers of motion. Those with $z$ values three or less (open bar) were not significantly different from a random distribution around a circle ( $z$ value for the neuron in $B$ was 0.2 ). Those with $z$ values greater than 6 (solid bars) were significantly different from random (with a few exceptions due to small sample size), suggesting a unimodal relationship between the center of motion and response amplitude ( $z$ value for the neuron in $A$ was 86 ). Those with $z$ values greater than 3 but 6 or less (shaded bar) were, or were not, significant as a function of sample size. A total of $65 \%$ of the neurons yielded significant $z$ values, indicating most had unimodal responses. 
A
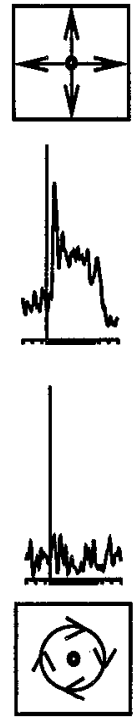

B
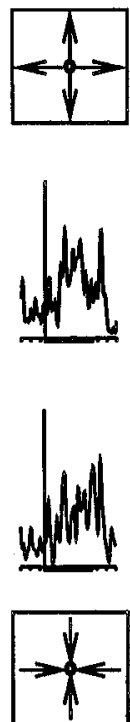

\section{SHIFTED CENTERS OF MOTION}
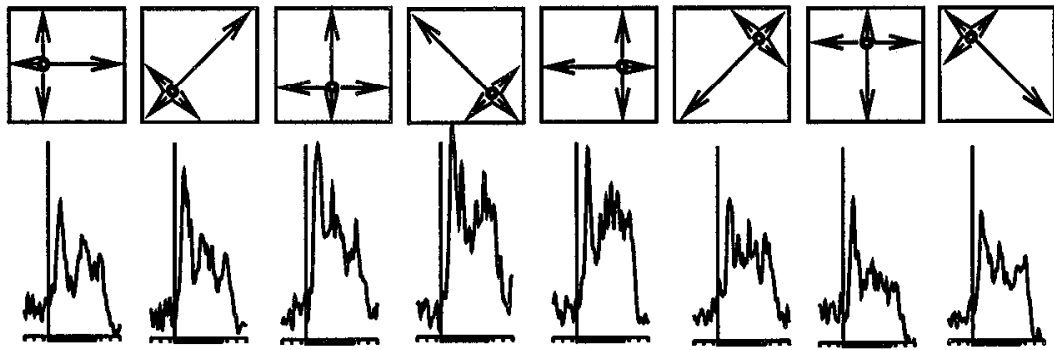

26KR18
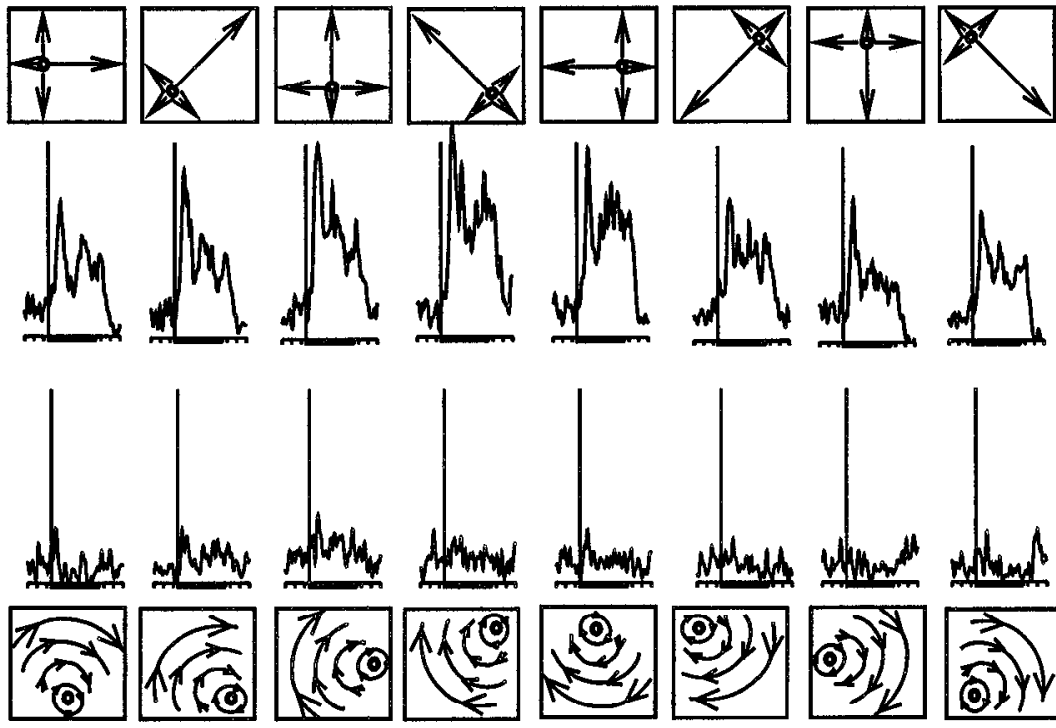

SHIFTED CENTERS OF MOTION
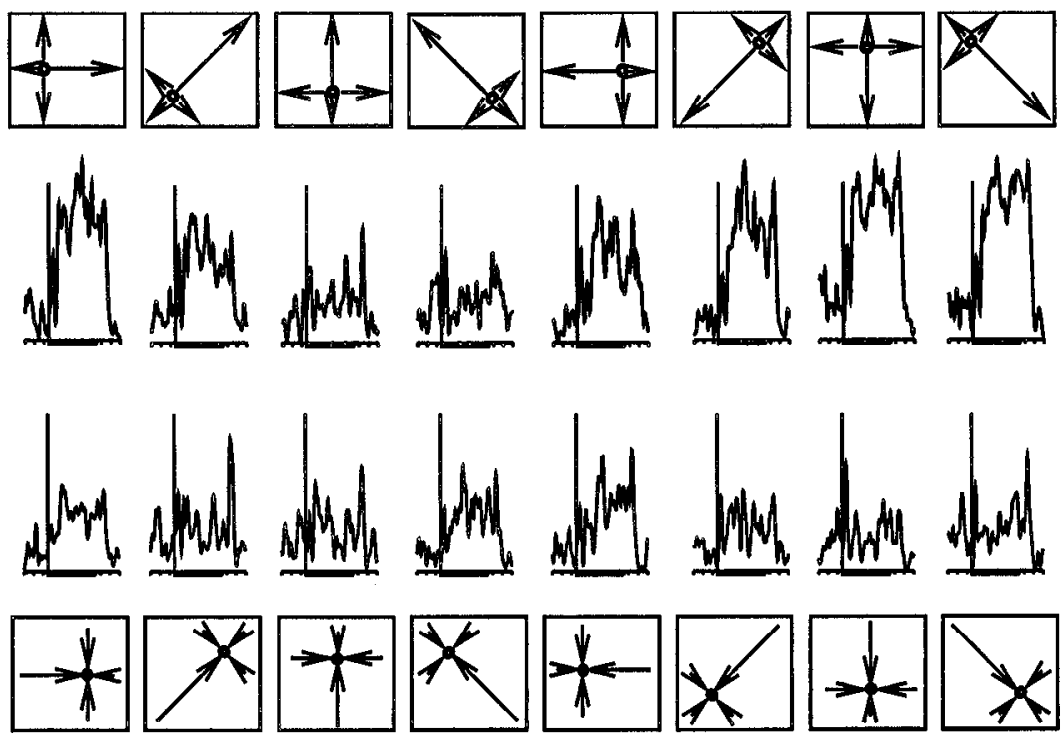

26KR14

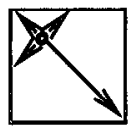

C

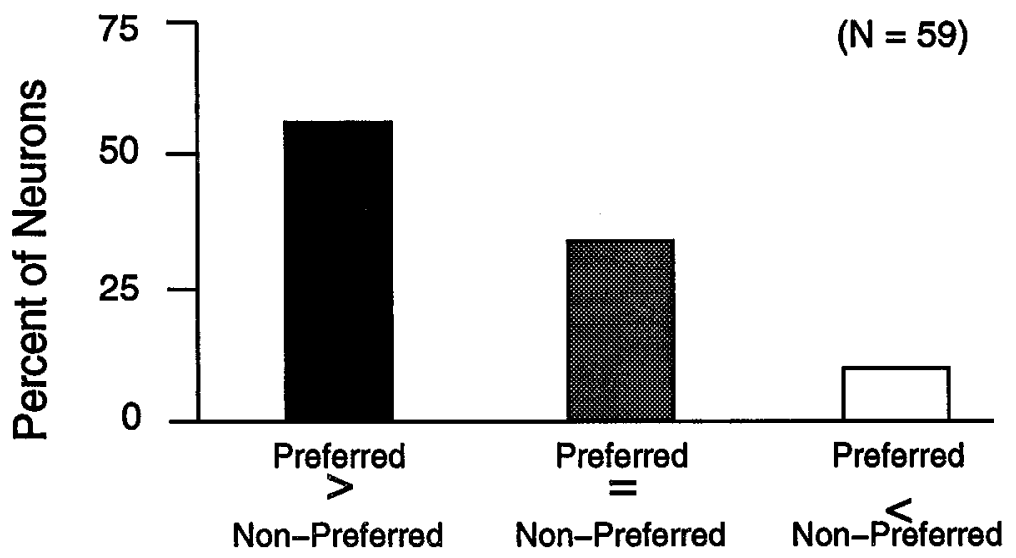

evoked stronger responses (Rayleigh $z$ for this distribution equals 10.55 , significant $p<0.001$ ) than the nonpreferred circular shifted center of motion stimuli $(z=6.12$, not significant). This illustrates the effect of stimulus preference for one type of motion (radial vs circular). This neuron's receptive field covered the left lower quadrant, and extended $15^{\circ}$ into the left upper quadrant. $B$, Comparison between the responses of a neuron that responded only somewhat better to outward radial stimuli (top row) than to inward radial slimuli (bottom row). The outward radial shifted center of motion stimuli evoked stronger responses $(z=37.42$, significant $p<0.001$ ) than the nonpreferred inward radial shifted center of motion stimuli $(z=1.90$, not significant). This illustrates the effect of stimulus preference for one direction of motion (outward vs inward) within a type of motion. This neuron's receptive field covered the left upper quadrant, and extended $10^{\circ}$ into the left lower quadrant. Vertical line on the spike density plots indicates 75 spikes/sec; horizontal bar indicales $1 \mathrm{sec}$. C, Comparison of responses to shifted centers of motion stimuli derived from preferred and nonpreferred stimuli for all 59 cells so studied. If the neuron showed a significantly stronger response ( $t$ test, $p<0.01$ ) to one of the preferred shifted centers of motion stimuli than to any of the nonpreferred shifted center of motion stimuli it was placed in the left bin of the histogram. Most neurons $(56 \%)$ showed stronger responses to the preferred stimuli with shifted centers of motion 

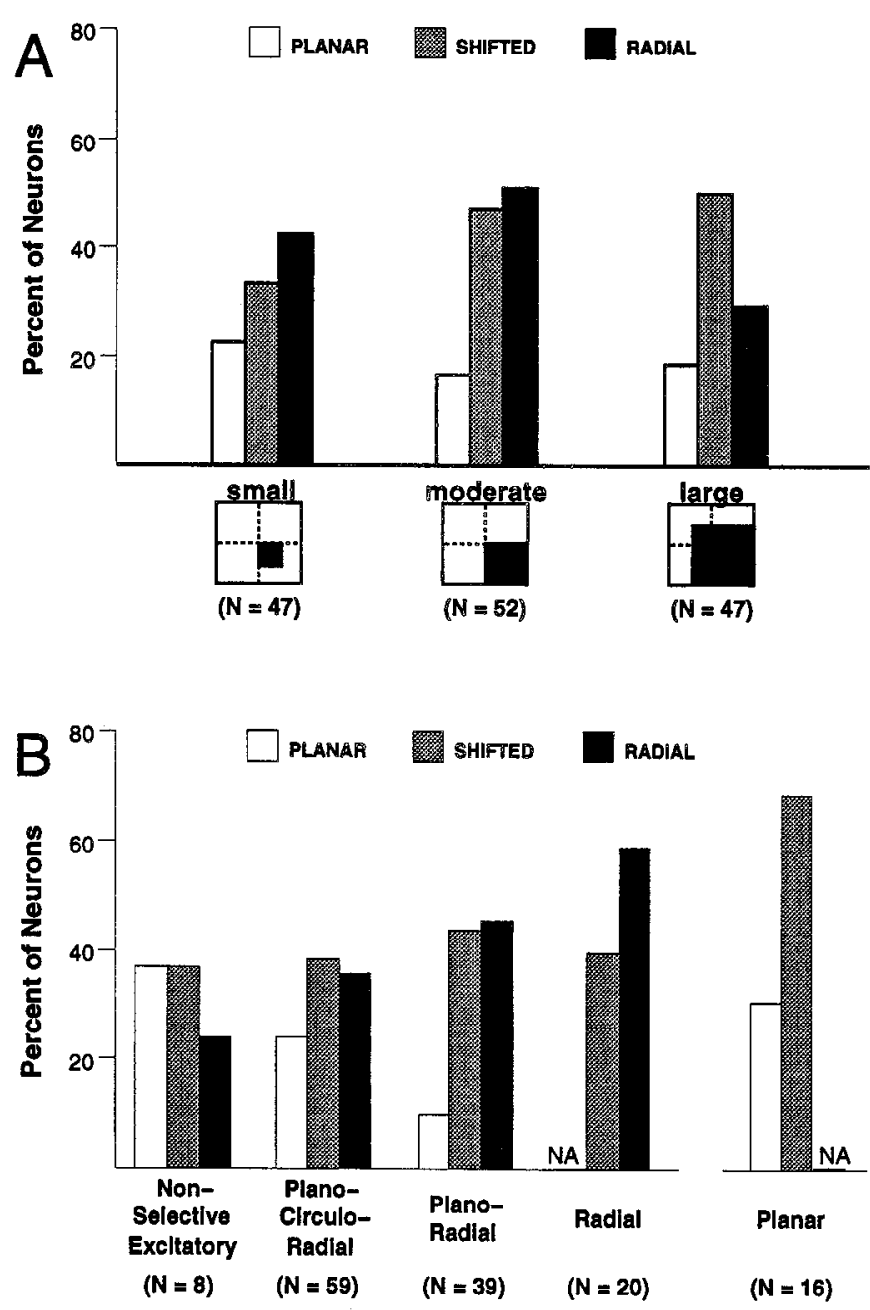

Figure 7. Fraction of neurons that gave the best response to shifted center of motion radial stimuli, to centered radial stimuli, or to planar stimuli for neurons with different receptive field sizes $(A)$ and different motion component classifications $(B)$. All neurons are included that responded best to radial motion and that were tested with radial shifted center of motion stimuli. $A$, Percent of neurons that gave the best response ( $t$ test, $p<0.01$ ) to planar, shifted, or centered stimuli for neurons having small (sides of $15-40^{\circ}$ ), moderate $\left(40-10^{\circ}\right)$, and large $\left(>70^{\circ}\right)$ receptive fields. The receptive field size was not uniquely related to a type of center of motion preference, and no clear trends are evident. $B$, Percent of neurons that gave the best response ( $t$ test, $p<0.01$ ) to planar, shifted, or center stimuli for neurons in each motion component category (single, double, or triple as in Fig. 3). The motion component category of a given neuron does not predict its response to the stimuli with shifted centers of motion.

the population might be capable of responding to a wide variety of different centers of motion.

The bar graphs in Figure 9, $B$ and $D$, show the proportion of these ncurons preferring central, eccentric, and peripheral centers of motion. The most frequently preferred stimuli with shifted centers of motion among both the radial and circular neurons were those with eccentric centers of motion. This preference included two neurons that showed no statistically significant response to any of the 12 basic motion stimuli (see Fig. 2) but showed strong responses to stimuli with eccentric centers of molion. Although the eccentric centers of motion were the most frequently preferred stimuli, this in part reflects the fact that eight center of motion stimuli are summed together. The most frequently preferred individual stimulus is the centered one for both the radial and circular stimuli. Thus, taking the mean number of neurons along a line from periphery to center, there was an increasing preference for centers of motion closer to the center of the field.

The higher frequency with which neurons responded best to a motion stimulus centered in the visual field could reflect the fact that the initial screening study presented only radial and circular stimuli with the center of motion in the center of the field. However, we studied all of the neurons we could successfully isolate, and we usually did not know into what component class the response to our standard 12 stimulus test set would place the neuron, a procedure that should have reduced the impact of any selection bias.

\section{Specificity for preferred centers of motion}

Because many neurons had significant responses to stimuli with centers of motion in more than one location, we measured how wide spread these preferred centers were by determining the number of centers of motion stimuli evoking responses that were not significantly different from the best response. In Figure 10 we divided the responses into highly selective (responses to one center of motion), moderately selective (two or three centers of motion), and less selective ( $>$ three centers of motion). A substantial number of neurons (19\%-45/236, solid bar in Fig. 10) were highly selective, and an additional $36 \%(85 / 236$ - shaded bars) were moderately selective so that over half of the neurons responded to centers of motion in no more than three locations. Over $95 \%$ of the neurons had significant responses to eight or fewer stimuli. We consistently found the centers of motion contiguous with one another rather than spread separately around the visual field.

One might expect that center of motion preference should possess greater spatial resolution towards the center of the visual field, providing observers with more refined information of subtle path deviations in the most common circumstances (looking where they are going). To test this prediction we determined whether the number of centers of motion stimuli to which a cell responded was related to where the preferred centers of motion were located. Figure 11 makes this comparison for all neurons (Fig. 11A) and for neurons having preferred centers of motion centered, eccentric, or peripheral in the field (Fig. $11 B-D$ ). The neurons preferring the center showed a greater percentage with a high degree of center of motion selectivity. The neurons preferring eccentric and peripheral centers of motion showed lower percentages with a high degree of center of motion selectivity.

This indication of greater spatial selectivity for centers of motion in the center of the field, prompted us to test neurons with a larger number of stimuli having centers of motion concentrated closer to the center of the field. To do so, we added a set of stimuli having centers of motion which were midway between those of the eccentric and centered stimuli $\left(22^{\circ}\right.$ from the center of the stimulus; Fig. 12A). Гigure $12, B$ and $C$, shows the results of such a study in a planoradial neuron that responded to inward radial motion. In this neuron, stimuli having centered or peripheral centers of motion evoked weak responses, eccentric stimuli evoked stronger responses, and pericentric stimuli evoked the strongest responses. Of the 44 neurons studied with this expanded set of 25 stimuli, half preferred pericentric stimuli, with the remainder distributed between peripheral, eccentric, and centered preferences. Thus, the more extended stimulus set revealed a more localized center of motion preference than could be seen with the set of only 17 stimuli and revealed a greater spatial 
A
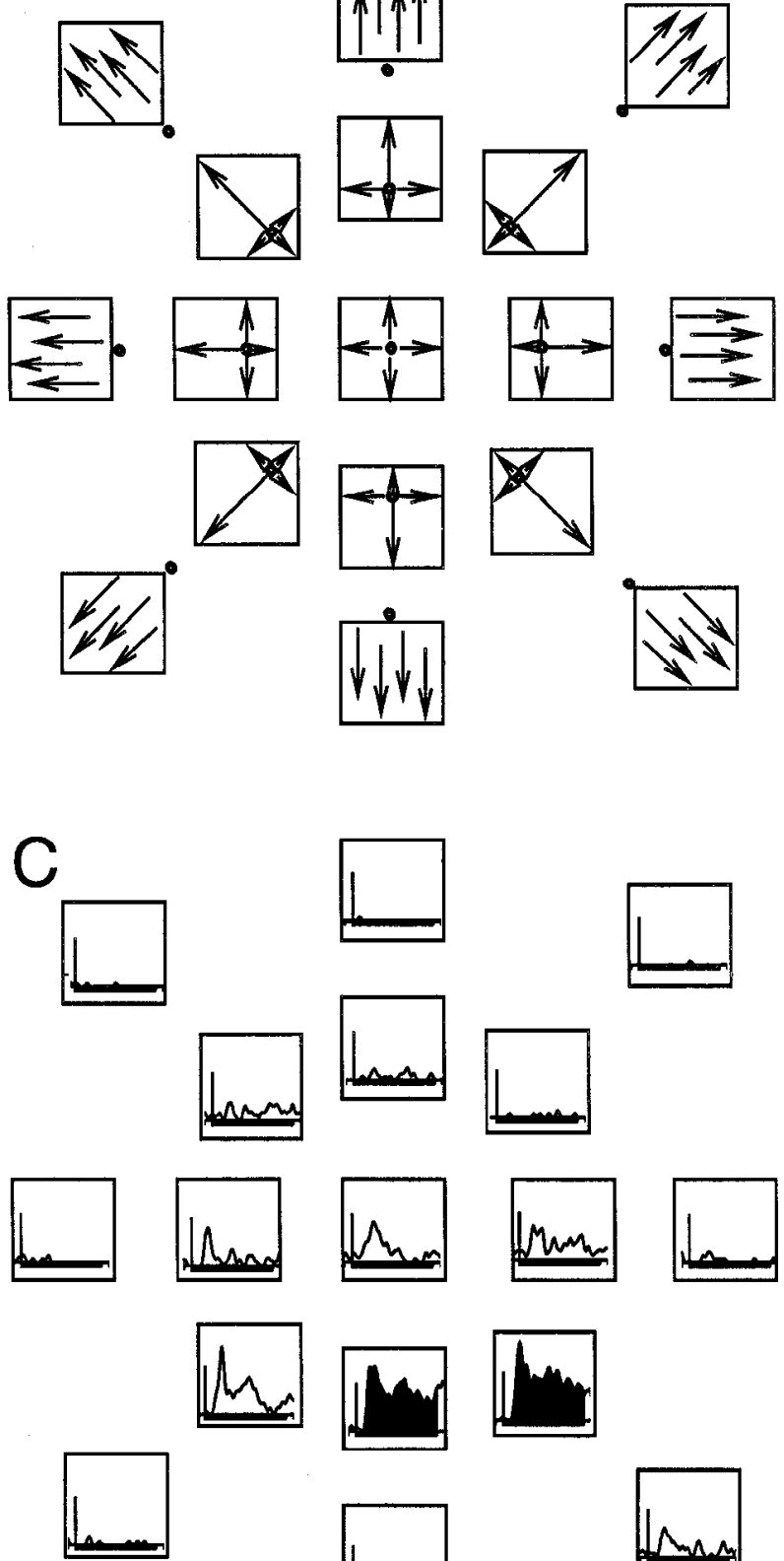
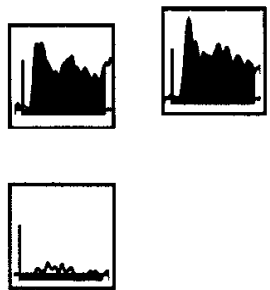
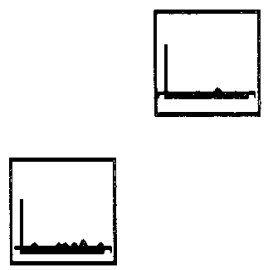

B
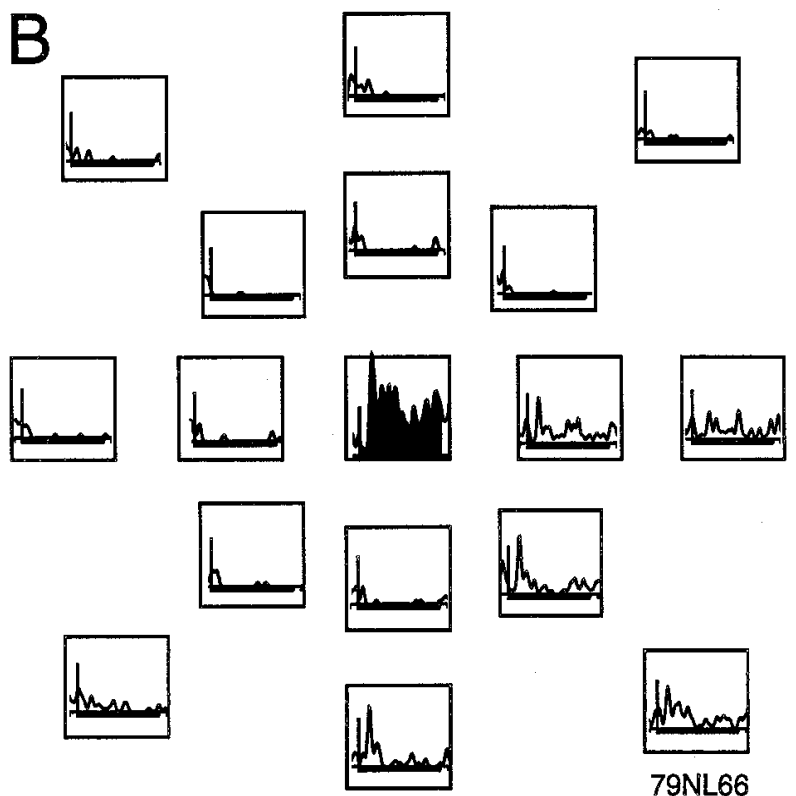

79NL66
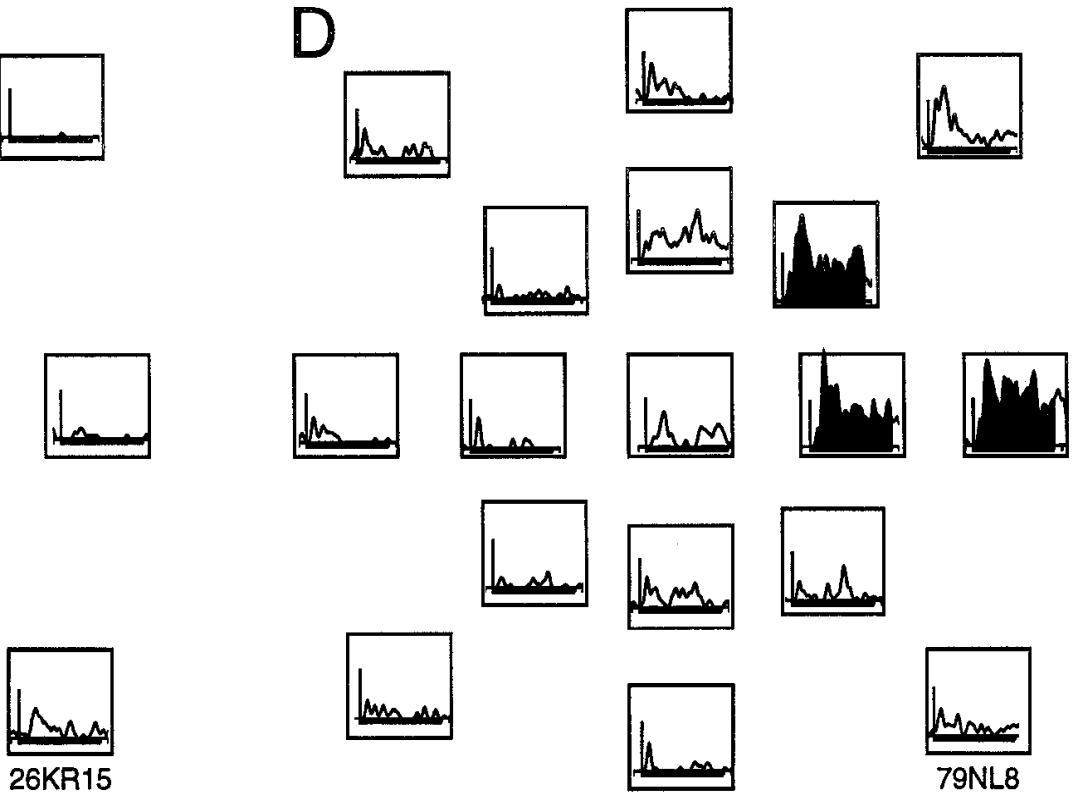

Figure 8. Location of the best responses to the center of motion stimuli. A, The 17 stimuli used with neurons that responded to outward radial motion. Each box represents the $100^{\circ} \times 100^{\circ}$ stimulus, the arrows within each box represent the directions of visual motion, and the circles indicate the center of motion. The outer ring of boxes represent planar stimuli; their outward centers of motion can be regarded as being $90^{\circ}$ from the center, off the screen in these stimuli. The inner ring of boxes represent stimuli with shifted centers of motion, their outward centers of motion are $45^{\circ}$ from the center, near the edge of the screen in these stimuli. The center box represents the outward radial stimulus with its center of motion at the center of the screen. $B-D$, Spike density histograms showing the responses of three neurons to the stimuli occupying the same positions as the stimuli illustrated in $A$. The darkened histograms are those that were not significantly different from the largest response $(t$ test, $p<0.01)$. $B$, A neuron that responded best to the stimulus with its center of motion at the center of the screen. This radial neuron's receptive field was in the right hemifield. $C$, A neuron that responded best to stimuli with eccentric centers of motion. This radial neuron's receptive field covered a $50^{\circ} \times 35^{\circ}$ area in the left upper quadrant. $D$, A neuron that responded best to a planar stimulus with a peripheral center of motion. This planoradial neuron's receptive field covered a $20^{\circ} \times 20^{\circ}$ area in the right upper quadrant.

resolution near the fixation point. Thus, the central area of the visual field appears to be represented by a relatively greater proportion of the neurons (Fig. 9) and by neurons that yield their best responses to centers of motion limited to the smallest area of the visual field (Fig. 11).

With the expanded set of stimuli (25) we could also measure how close together the preferred stimuli were in the visual field. For example, Figure $12 \mathrm{C}$ shows that the stimuli that evoked responses comparable to the best response (solid) or significantly above control activity level (shaded) were clustered together in the visual field. This illusirates a clustering of the centers of motion that evoke strong responses from a neuron that 

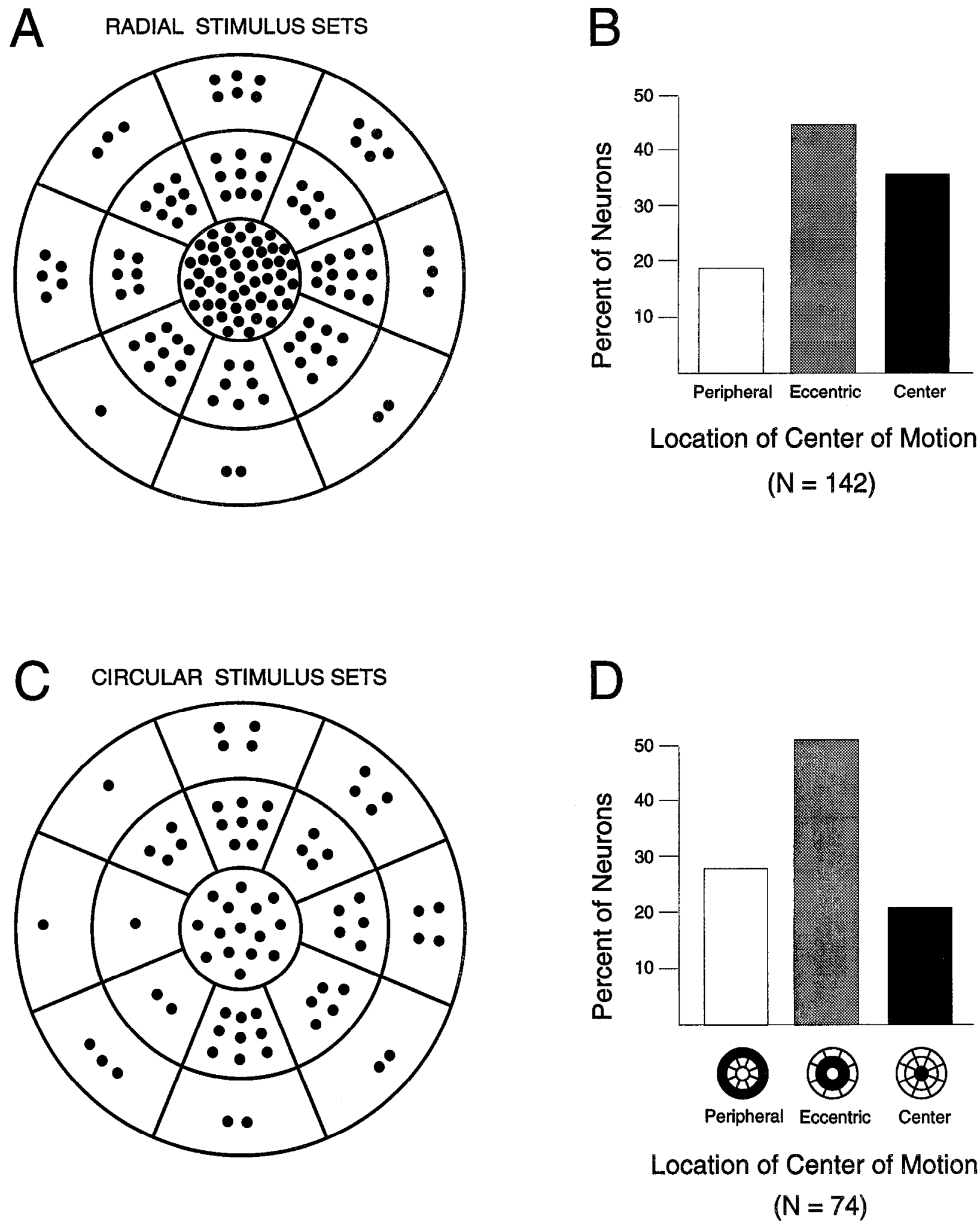

Figure 9. Distribution of centers of motion that evoked the strongest response. A, Distribution for neurons that preferred radial (inward or outward) stimuli. The 17 segments of the circular plot represent the 17 stimuli, with each segment representing the center of motion stimulus depicted at the corresponding position in Figure 8A. Each dot represents a neuron, and the dots are placed in the segment which corresponds to the stimulus that evoked the largest response from that neuron. All tested centers of motion are preferred by at least one neuron. $B$, The relative distribution of the preferred centers of motion shown in $A$. The graph shows the percentage of neurons that had the strongest response to center, eccentric, or peripheral centers of motion stimuli. Stimuli having eccentric centers of motion were most commonly preferred, but the stimulus with the center of motion in the center of the screen was the single stimulus preferred by the largest number of neurons. $C$, Distribution for neurons that preferred circular (clockwise or counterclockwise) stimuli. Again, all centers of motion are preferred by at least one neuron. $D$, The relative distribution of the preferred centers of motion shown in $C$. $\Lambda$ s with the neurons tested with radial combinations, eccentric centers of motion are most commonly preferred, but the centered center of motion stimulus was the single stimulus preferred by the greatest number of neurons. 


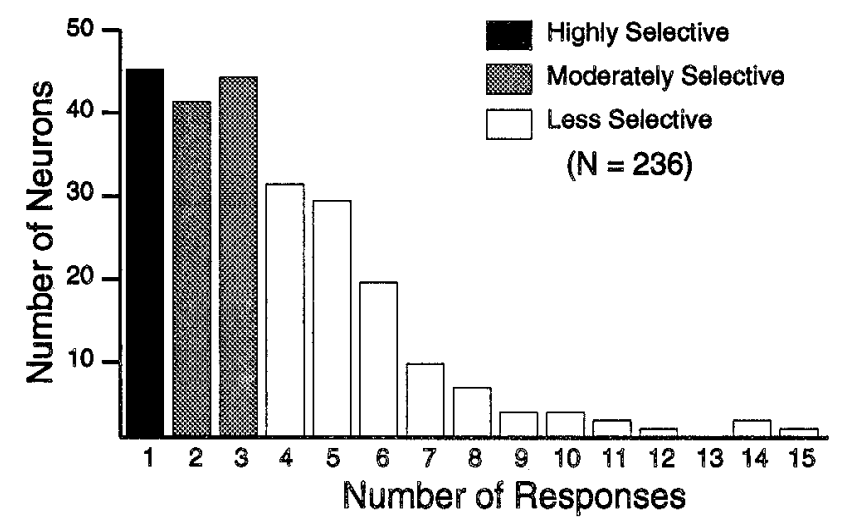

Figure 10. Number of the 17 stimuli evoking a significant response in neurons studied with radial and circular stimuli with shifted centers of motion. Neurons are divided into three groups: highly selective neurons (solid bar) in which only one of the 17 stimuli evoked a response significantly different from the background response, moderately selective neurons (shaded bars) in which two or three stimuli evoked significant responses, less selective neurons (open bars) in which more than three stimuli evoked significant responses. Over half the neurons responded to centers of motion in no more than three locations.

was evident in all of our experiments. In the 44 neurons studied with the 25 stimuli, $97 \%$ of the 233 significant responses were adjacent to other significant responses. Thus, the preferred stimuli lie in one part of the visual field.

We examined the spatial distribution of the preferred centers of motion in more detail in 12 neurons in which we presented 33 stimuli having centers of motion within the central $40^{\circ}$ of the visual field, arranged at $10^{\circ}$ intervals along four axes: horizontal, vertical, and both $45^{\circ}$ obliques (Fig. 13A). Figure $13 B-D$ shows the results of studies on three neurons. The neuron in Figure $13 B$ had its strongest responses to stimuli having centers of motion in the lower left corner of the visual field. The neuron in Figure $13 \mathrm{C}$ had its strongest responses to stimuli having centers of motion clustered around or at the center of the visual field or symmetrically around it. The neuron in Figure $13 D$ had it strongest responses to stimuli having centers of motion asymmetrically clustered around the center of the field, but with a notch of decreased responsiveness to stimuli with the center of motion at the center of the visual field. The three examples illustrated in Figure 13 represent the three major types of spatial structure observed in these 12 neurons: a peripheral corner (5 neurons), a central peak (4 neurons), and a central notch (3 neurons). These studies suggest that the preferred center of motion, rather than being a simple segment of the visual field, may have a more complex structure which our relatively limited stimuli have not fully defined.

Figure 11. Interactions between the location of the preferred center of motion (as in Fig. 9) and the degree of center of motion selectivity (as in Fig. 10). A, The percentage of all neurons tested which showed highly (solid bar), moderately (shaded bar), or less (open bar) selective responses for center of motion stimuli. $B-D$, The percentages of center preferring $(B)$, eccentric preferring $(C)$, and peripheral preferring $(D)$ neurons which showed highly, moderately, or less selective responses for center of motion stimuli. Center preferring neurons most commonly
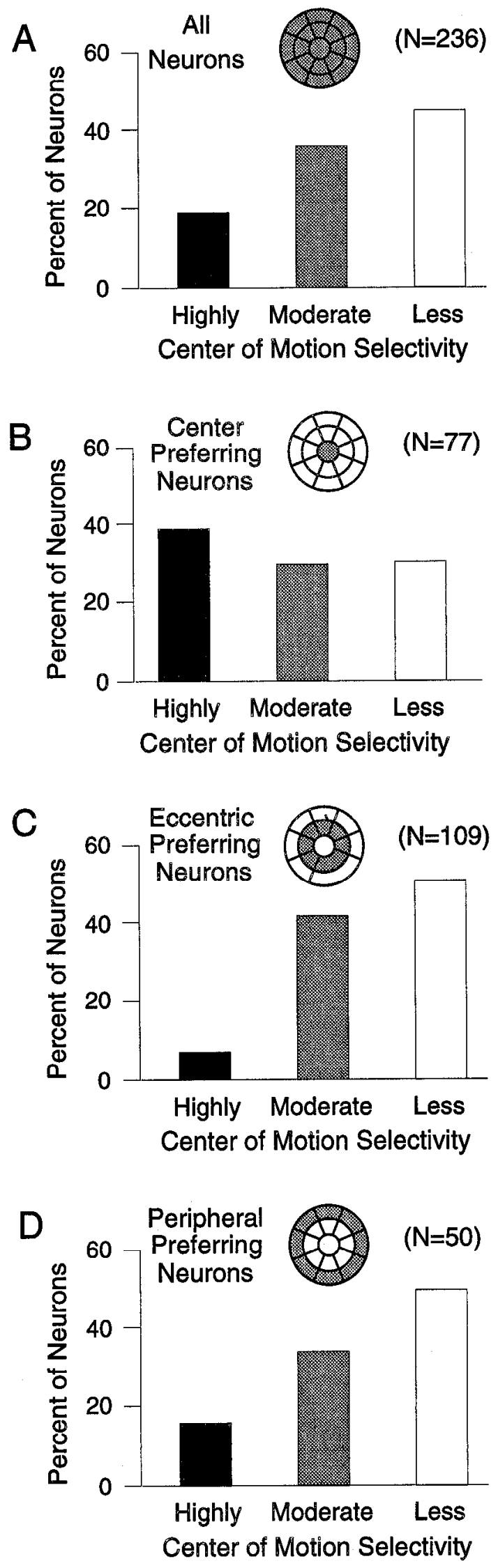

showed highly selective responses, whereas eccentric and peripheral preferring neurons most commonly showed moderately or less selective responses. 

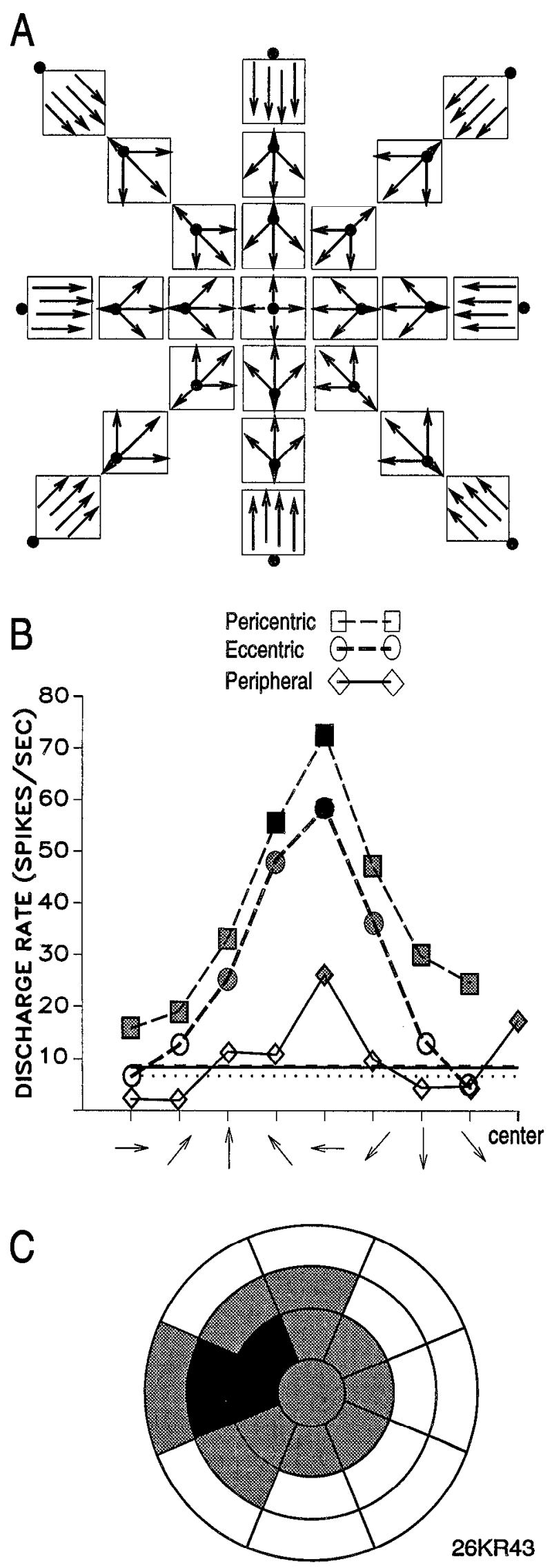

Figure 12. Responses of a neuron to an extended set of center of motion stimuli. $A$, Schematic representations of 25 inward radial center of motion stimuli, including 8 added pericentric center of motion stim-

\section{Discussion}

Neuronal responses to shifted centers of motion

We studied MSTd neurons that responded to radial or circular motion in the middle of the visual field by presenting the preferred pattern with its center shifted to eight points around the field. We found that MSTd neurons were sensitive to such shifts: at least one of the eight shifted center stimuli produced a response significantly different from the centered stimulus in $91 \%$ of the neurons. In our sample of 245 neurons, over half gave significant responses to three or fewer stimuli; the preferred centers of motion were clustered in one part of the field and not scattered about. The neurons studied preferred centers of motion throughout all parts of the visual field; a larger number of neurons preferred the center of motion in the middle of the field and these neurons responded to centers of motion over a more limited area of the visual field. These observations suggest that the shifted center of motion, and the accompanying changes in the pattern of motion throughout the visual field, is a stimulus characteristic which influences the responses of MSTd neurons.

\section{Hypothesis on functional organization}

Based on these observations, we suggest that each of the MSTd neurons can be regarded as having a center of motion field. The responses of individual neurons would be graded according to the proximity of the center of motion of a stimulus to the preferred center of motion for the neuron, just as other visual cortical neurons have graded preferences for the direction of planar motion or for the location of a spot of light. The role of MSTd neurons in interpreting optic flow fields would not be one of qualitative feature matching, but rather one of responding to visual motion according to the degree of match between the visual input and the preferred optic flow field of the neuron. Thus, each neuron would contribute to the population response to a number of stimuli, just as many neurons would contribute to the population response evoked by any stimulus. This population encoding would have the advantage of remaining robust in spite of idiosyncracies in any individual neuron's response profile.

We suggest further that there is an orderly arrangement of MSTd neurons with each region of the visual field being represented by a set of neurons whose center of motion falls in that region of the field. The population of neurons would have a gradient in the density of the centers of motion with those near

$\leftarrow$

uli. The pericentric centers of motion are shifted $22^{\circ}$ from the center of the screen, halfway between the eccentric centers of motion $\left(45^{\circ}\right)$ and the screen center. $B$, Graph of response amplitudes evoked by stimuli shown in $A$ with peripheral (diamonds), eccentric (circles), pericentric (squares), and centered (far right diamond) centers of motion. Shaded symbols indicate responses that were significantly above control activity level, solid. symbols represent responses that were not significantly different from the best response. The horizontal lines indicate unstimulated control activity in the trials whose responses are represented by the same type of line. Arrows on abscissa show the direction of the shift of the center of motion. $C$, Plot of these responses with 25 segments representing the 25 center of motion stimuli arranged as shown in $A$. The shaded and solid segments show the same significant responses shown in $B$. Stimuli having centers of motion immediately to the right of the eenter of the sereen evoked the strongest response which indicates a finer grain in the preferred center of motion location than can be determined by stimuli with 17 centers of motion in Figure 8. This planoradial neuron's receptive field included all of the lower henifield, except the $10^{\circ}$ at the right most edge of the screen. 
A

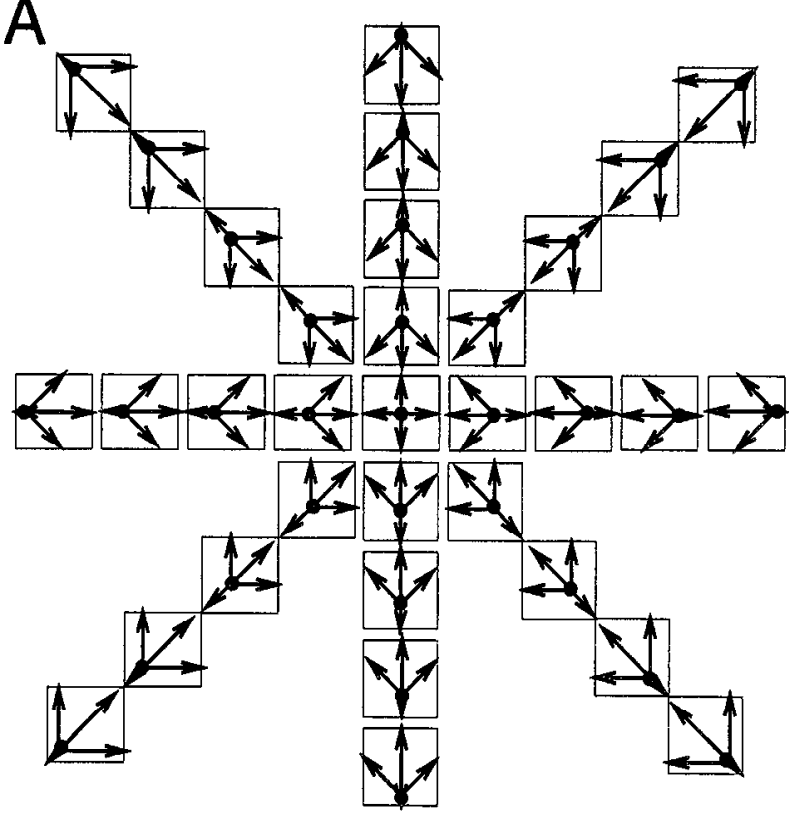

C

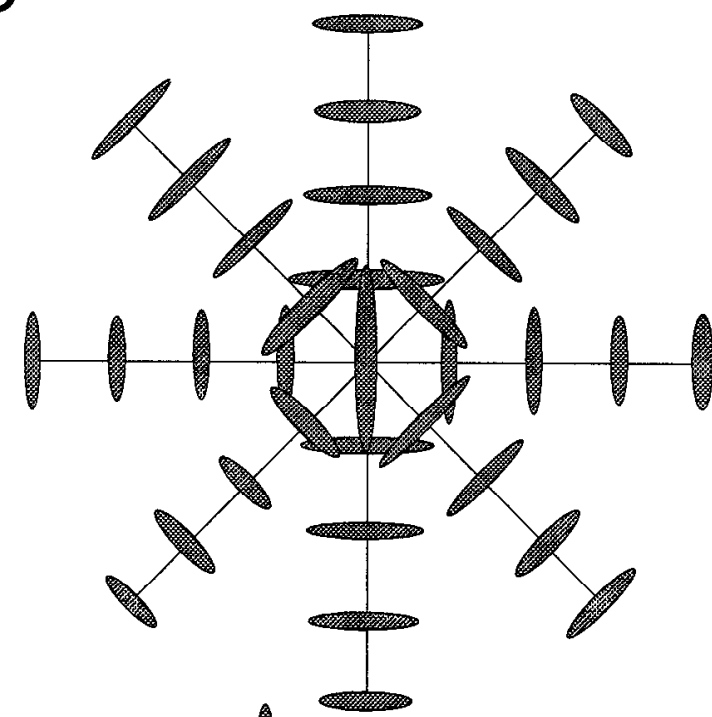

Control Activity
B

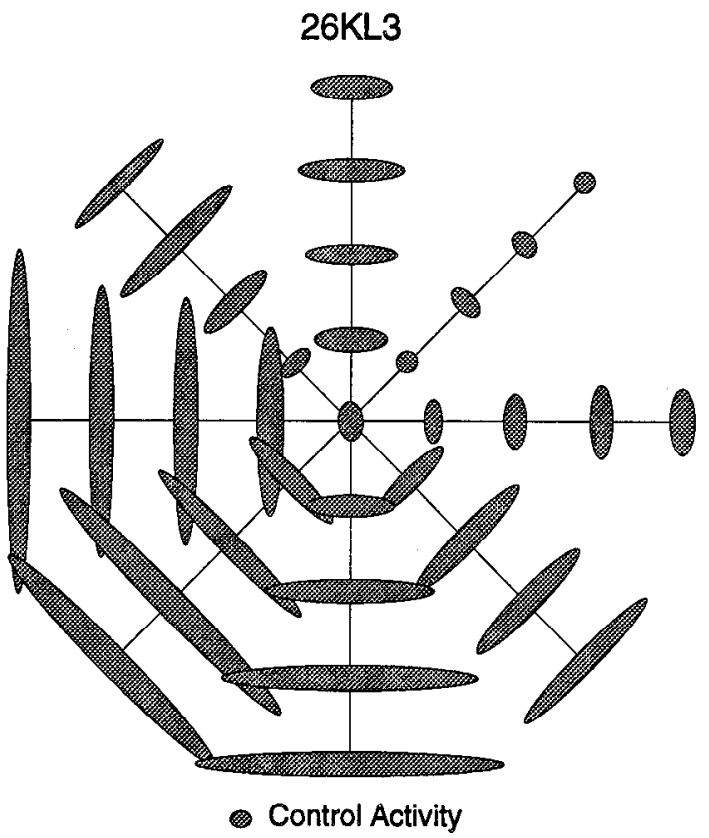

D

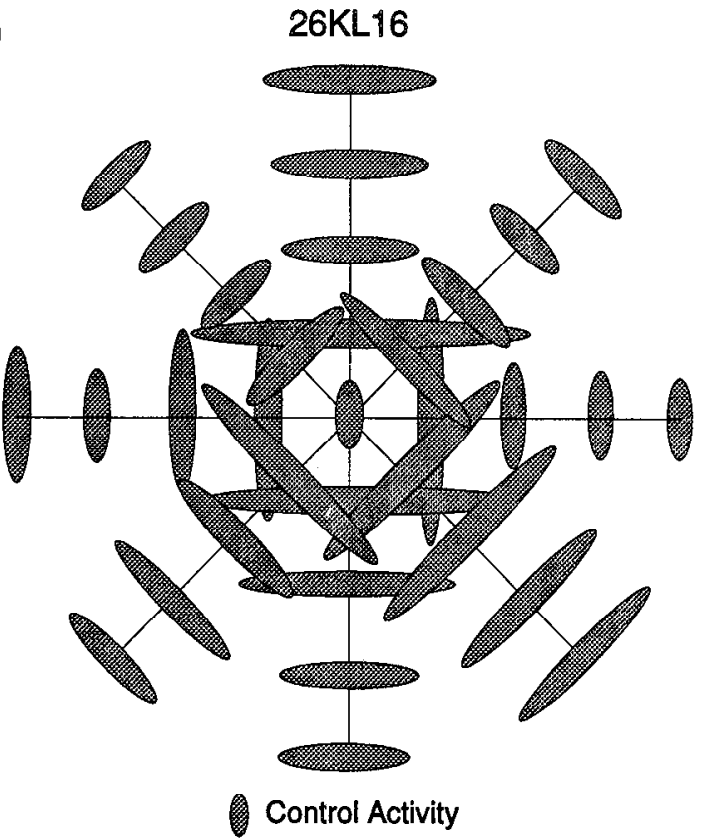

Figure 13. Types of neuronal responses to stimuli having centers of motion concentrated within the central $40^{\circ}$ of the visual field. $A$, Diagrammatic representations of a set of 33 outward radial stimuli. The centers of motion are distributed at $10^{\circ}$ intervals along four axes. $B-D$, The responses of three individual neurons. Responses are represented by ovals at positions which correspond to the relative position of the centers of motion. Response amplitude at each position is proportionate to the length of the oval and is derived from the spike density histograms as described in Materials and Methods. Activity during unstimulated control trials is represented below each plot. $B$, The responses of a neuron that preferred stimuli having centers of motion in the lower left corner of the screen, with decreasing response amplitude graded towards the center. This planoradial neuron's receptive field covered the right hemifield to $30^{\circ}$ above and below the horizontal meridian. $C$, The responses of a neuron that preferred stimuli having centers of motion nearer the center of the screen, with the greatest responses to stimuli in which the center of motion was at the center of the screen. This radial neuron's receptive field covered the entire central $100^{\circ} \times 100^{\circ}$ area of the projection screen. $D$, The responses of a neuron that preferred stimuli having centers of motion nearer the center of screen, but with a smaller response to stimuli in which the center of motion was at the center of the screen. This radial neuron's receptive field extended for $40-45^{\circ}$ from the fixation point into the right lower quadrant. 


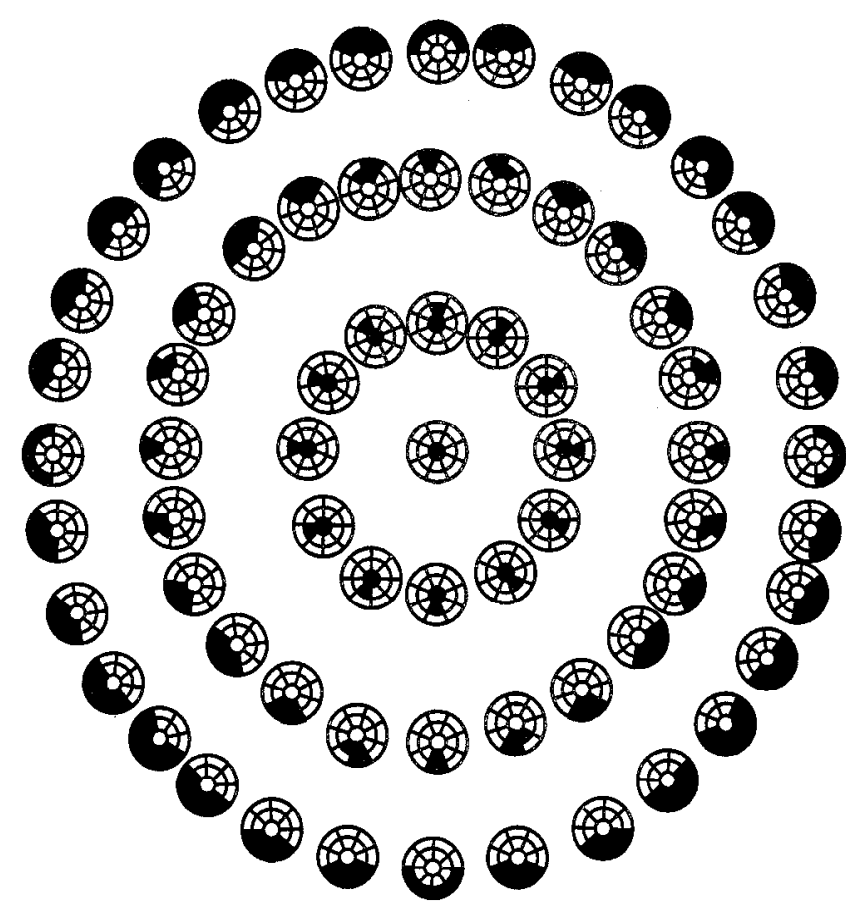

Figure 14. Hypothesized map for preferred centers of motion across neurons in MST. Darkened area in each circle indicates the area of the field to which a neuron is sensitive. The schematic drawing is based on the key observations that all areas of the visual field are represented by some neurons, and that there is a gradient of sensitivity for centers of motion, with those neurons responding to more peripheral centers of motion, rcsponding to such centers over a larger part of the field.

the middle of the visual field more highly represented and responding to centers of motion over a more limited area of the visual field. Figure 14 shows this hypothesis schematically for the neurons responding to radial motion; such an arrangement of neurons might exist for circular motion as well. While an orderly anatomical arrangement remains to be established, an organization based on this functional criteria of centers of motion would be an interesting shift in organization from the retinotopic one of the adjacent MT area.

\section{Relation to heading determinations}

We think that MSTd neurons that respond to radial motion with centers of motion (or a focus of expansion (FOE) in the case of the radial stimulus) arrayed across the visual field could contribute to the determination of heading of observers moving through the environment. While heading maybe determined by a combination of visual cues (Cutting, 1986), our findings support the conclusions of a number of recent psychophysical experiments suggesting the existence of brain mechanisms which might derive heading from optic flow. Recent psychophysical experiments using simulations of the optic flow produced by observer movement have demonstrated that humans can make heading judgements within a few degrees of the simulated heading (Warren and Hannon, 1988, 1990; Warren et al., 1988; Stone and Perrone, 1991, 1993; van den Berg 1992). Such judgements are frequently better when the eyes move (Royden et al., 1992) and fail when only a single plane (like a wall) is present (Regan and Beverley, 1982; Warren and Hannon, 1990).

In our physiological experiments, we have tried to eliminate all cues but optic flow, simulating motion toward a single plane during visual fixation of a stationary target. The information relevant to heading judgements in our display therefore is limited. Our physiological observations would be entirely relevant, however, to determining heading in the circumstance in which the point of fixation is at a distance, without the interaction with multiple depth planes or pursuit eye movements. The forward view from an airplane during landing, used by Gibson (1986) to illustrate flow patterns, would seem to closely approximate this condition since there is radial flow with little rotation. For example, the MSI'd neurons studied with the dense array of center of motion stimuli (Fig. 13) revealed patterns of center of motion sensitivity which suggests that some cells might differentiate between going where the observer is looking versus going in another direction. This is illustrated by the comparison of Figure $15, A$ and $B$, in which the flight path leading towards the landing strip (Fig. 15A) would be detected by a neuron with a preferred center of motion in the center of the field whereas a flight path leading to the lower left corner (Fig. 15B) would be detected by a neuron with the preferred center of motion off to the side. While this is a limited and highly specific example of the use of the FOE structure of these MSTd cells, they indicate the type of heading information that could be provided by the neurons.

Our experiments might also be relevant to the recent models which attempt to show how directionally selective elements might produce heading judgements (Heeger and Jepson, 1990; Warren et al., 1991; Perrone, 1992; Lappe and Rauschecker, 1993; Perrone and Stone, 1994). All attempt to use two-dimensional retinal information to reconstruct three-dimensional heading information, with some requiring differential motion in a region of the field (Longuet-Higgins and Prazdny, 1980; Rieger and Lawton, 1985; Rieger and Toet, 1985). In general, determination of directionally selective elements in the models corresponds to neuronal activity in MT whereas the elements related to heading are more comparable to the neurons studied in MSTd. However, we believe that our data do not allow us to definitively favor any of these hypotheses.

Our hypothesis of a map of headings among the MSTd neurons is particularly relevant to the recent models of Perrone (1992) and of Perrone and Stone (1994), who used a template to determine heading: a map of the visual field has different headings represented by different neural elements, and the heading is identified by the most active element. The organization of heading directions that we have hypothesized (Fig. 14) is almost identical to the heading map shown in this model. In addition, the series of constraints that they place on this model to make it biologically realistic (Perrone and Stone, 1994) produces an organization that would seem to have the same grain of heading specification suggested by our experiments. Further specification of the density of the hypothesized heading map in MSTd and the effect on this map of rotation in addition to translation might allow further convergence between the neural models and the physiological hypotheses.

\section{Position invariance}

A key point in the analysis of MST neuronal responses to the components of optic flow stimuli is whether these responses can simply result from planar motion in one part of their receptive fields. In our previous experiments (Duffy and Wurtz, 1991a), we addressed this issue by testing whether radial or circular stimuli, which were effective when presented over the full $100^{\circ}$ $\times 100^{\circ}$ field, remained effective when we presented a smaller version of the same stimulus. We found that the single component radial and circular neurons were the most likely to continue 

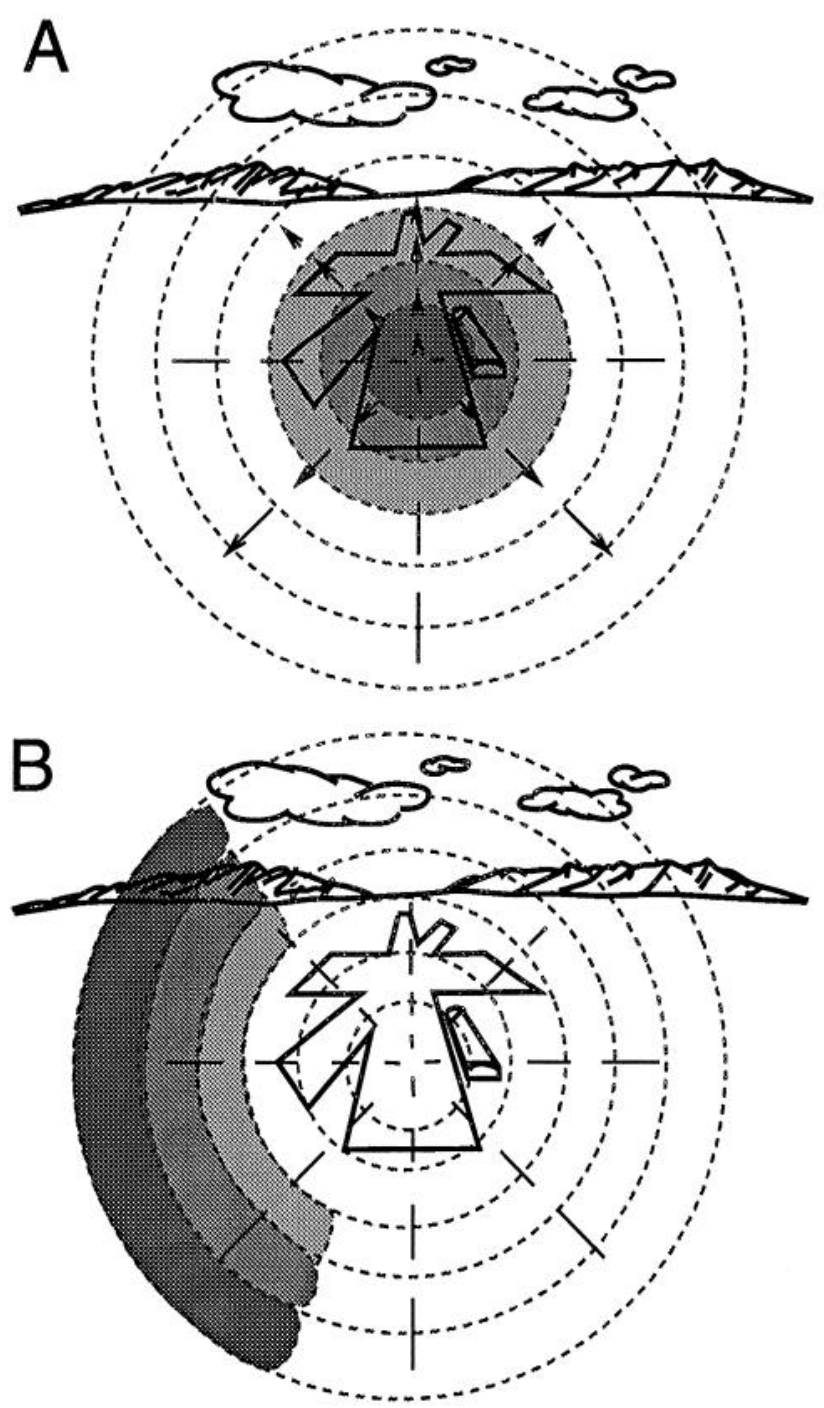

Figure 15. An example of the potential application of elements in the map of center of motion preference. In this illustration, the observer is utilizing optic flow in piloting an approach to an airport (drawings after Gibson, 1986). The observer's field of view is represented by a series of concentric circles to mark eccentricity. The shaded areas within these fields represent regions of center of motion responsiveness of MSTd neurons, with darker shading depicting subregions of greater responsiveness. These idealized response fields reflect findings illustrated in Figure 13. $A$, A peaked pattern neuron would respond best when the center of motion is over the fixation target on the landing strip when the pilot is looking where he is going. $B$, A graded pattern neuron would respond best when the center of motion is in the periphery, to the left of the landing strip.

to respond as we moved these small field stimuli into different segments of the larger field, indicating that their responses could not be explained by their response to simple planar motion. In contrast, triple component neurons showed the greatest change in response when the stimuli were moved to different regions of the field suggesting that their responses could result from sensitivity to simple planar motion. Andersen et al. (1990, 1993) and Graziano et al. (1994) used smaller stimuli $\left(10^{\circ} \times 10^{\circ}\right)$ and shifted them $20^{\circ}$ to study the same issue, and also found many MST neurons that continued to respond after the stimulus was shifted. Orban and his collaborators (Orban et al., 1991; Lagae et al., 1994) have reported a series of experiments which extend these conclusions and suggest a role for adjacent area MT in developing these response properties. In net, these experiments reach the same conclusion, that some MST neurons continue to respond when the stimulus is shifted on their receptive fields.

The continued response of some MST neurons in spite of a shift of stimulus location has been referred to as position invariance, and it has recently been argued (Graziano et al., 1994) that such position invariance would preclude the use of these neurons in the determination of heading. Our present experiments using large field stimuli, rather than the small field stimuli just discussed, reveal the limitations of position invariance. We found many cells that responded best when the center of motion fell in one region of the visual field, rather than another, and that about $90 \%$ of the MSTd neurons showed an alteration in the strength of their responses when the center of motion was shifted. When we look at the results of the previous experiments using small field stimuli, we also see shifts in the magnitude of the response even though the preferred stimulus remained the same, so that many of these observations would be better regarded as indicating relative invariance rather than absolute invariance. In the previous experiments, stimuli were shifted over relatively small distances in the field, leading to response changes that could now be explained by movement across the graded preference for centers of motion revealed by our large field stimuli. Thus, MSTd neurons could provide information related to heading.

\section{References}

Andersen RA, Snowden RJ, Treue S, Graziano M (1990) Hierarchical processing of motion in the visual cortex of monkey. Cold Spring Harbor Symp Quant Biol 55:741-748.

Andersen RA, Treue S, Graziano M, Snowden RJ, Quan N (1993) From direction of motion to patterns of motion: hierarchies of motion analysis in the visual cortex. In: Brain mechanisms of perception and memory: from neuron to behavior (Ono T, Squire LR, Raichle ME Perrett D, Fukuda M, eds), pp 183-199. New York: Oxford UP.

Batschelet E (1981) Circular statistics in biology. New York: Academic.

Crist CF, Yamasaki DSG, Komatsu H, Wurtz RH (1988) A grid system and a microsyringe for single cell recording. J Neurosci Methods 26: $117-122$.

Cutting JE (1986) Perception with an eye for motion. Cambridge: Bradford.

Desimone R, Ungerleider LG (1986) Multiple visual areas in the caudal superior temporal sulcus of the macaque. J Comp Neurol 248: 164-189.

Duffy CJ, Wurtz RH (1991a) Sensitivity of MST neurons to optic flow stimuli. I. A continuum of response selectivity to large-field stimuli. J Neurophysiol 65:1329-1345.

Duffy CJ, Wurtz RH (1991b) Sensitivity of MST neurons to optic flow stimuli. II. Mechanisms of response selectivity revealed by small-field stimuli. J Neurophysiol 65:1346-1359.

Duffy CJ, Wurtz RH (1991c) MSTd neuronal sensitivity to heading of motion in optic flow fields. Soc Neurosci Abstr 17:441.

Duffy CJ, Wurtz RH (1993) MSTd neuronal responses to the center of motion in optic flow fields. Soc Neurosci Abstr 19:1283.

Gallyas F (1979) Silver staining of myelin by means of physical development. Neurol Res 1:203-209.

Gibson JJ (1950) The perception of the visual world. Boston: Houghton Mifflin.

Gibson JJ (1986) The ecological approach to visual perception. London: Erlbaum.

Graziano M, Andersen R, Snowden R (1990) Stimulus selectivity of neurons in macaque MST. Soc Neurosci Abstr 16:6.

Graziano MSA, Andersen RA, Snowden R (1994) Tuning of MST neurons to spiral motion. J Neurosci 14:54-67.

Hays AV, Richmond BJ, Optican LM (1982) A UNIX-based multiple process system for real-time data acquisition and control. WESCON Conf Proc 2:1-10. 
Heeger DJ, Jepson A (1990) Visual perception of three-dimensional motion. Neural Comput 2:129-137.

Judge SJ, Richmond BJ, Chu FC (1980) Implantation of magnetic search coils for measurement of eye position: an improved method. Vision Res 20:535-538.

Komatsu H, Wurtz RH (1988) Relation of cortical areas MT and MST to pursuit eye movements. I. Localization and visual properties of neurons. J Neurophysiol 60:580-603.

Lagae L, Maes H, Raiguel S, Xiao D-K, Orban GA (1994) Responses of macaque STS neurons to optic flow components: a comparison of areas MT and MST. J Neurophysiol 71:1597-1626.

Lappe M, Rauschecker JP (1993) A neural network for the processing of optic flow from ego-motion in man and higher mammals. Neural Comput 5:374-391.

Longuet-Higgins HC, Prazdny K (1980) The interpretation of a moving retinal image. Proc R Soc Lond [Biol] 208:385-397.

MacPherson JM, Aldridge JW (1979) A quantitative method of computer analysis of spike train data collected from behaving animals. Brain Res 175:183-187.

Orban GA, Lagae L, Verri A, Xiao D, Raiguel S, Maes H (1991) Limitations of the optic flow selectivity in MST neurons. Soc Neurosci Abstr 17:525.

Orban GA, Lagae L, Verri A, Raiguel S, Xiao D, Maes H, Torre V (1992) First-order analysis of optical flow in monkey brain. Proc Natl Acad Sci USA 89:2595-2599.

Perrone JA (1992) Model for the computation of self-motion in biological systems. J Opt Soc Am A 9:177-194.

Perrone JA, Stone LS (1994) A model of self motion estimation within primate extrastriate cortex. Vision Res, in press.

Regan D, Beverley KI (1982) How do we avoid confounding the direction we are looking and the direction we are moving? Science 215:194-196.

Rieger JH, Lawton DT (1985) Processing differential image motion. J Opt Soc Am A 2:354-359.

Rieger JH, Toet $\mathrm{L}$ (1985) Human visual navigation in the presence of 3-D rotations. Biol Cybernet 52:377-381.

Robinson DA (1963) A method of measuring cyc movement using a scleral search coil in a magnetic field. IEEE Trans Biomed. Eng 10: $137-145$

Royden CS, Banks MS, Crowell JA (1992) The perception of heading during eye movements. Nature 360:583-585.

Saito H (1993) Hierarchical neural analysis of optical flow in the macaque visual pathway. In: Brain mechanisms of perception and mem- ory: from neuron to behavior (Ono T, Squire LR, Raichle ME, Perrett D, Fukuda M, eds), pp 121-140. New York: Oxford UP.

Saito H-A, Yukie M, Tanaka K, Hikosaka K, Fukada Y, Iwai E (1986) Integration of direction signals of image motion in the superior temporal sulcus of the macaque monkey. J Neurosci 6:145-157.

Stone LS, Perrone JA (1991) Human heading perception during combined translational and rotational self-motion. Soc Neurosci Abstr 17: 847.

Stone LS, Perrone JA (1993) Human heading perception cannot be explained using a local differential motion algorithm. Invest Ophthalmol Vis Sci 34:1229.

Tanaka K, Saito H (1989) Analysis of motion of the visual field by direction, expansion/contraction, and rotation cells clustered in the dorsal part of the medial superior temporal area of the macaque monkey. J Neurophysiol 62:626-641.

Tanaka K, Hikosaka K. Saito H-A, Yukie M, Fukada Y, Iwai E (1986) Analysis of local and wide-field movements in the superior temporal visual areas of the macaque monkey. J Neurosci 6:134-144.

Tanaka K, Fukada Y, Saito H (1989) Underlying mechanisms of the response specificity of expansion/contraction and rotation cells in the dorsal part of the medial superior temporal area of the macaque monkey. J Neurophysiol 62:642-656.

Ungerleider LG, Desimone R (1986) Cortical connections of visual area MT in the macaque. J Comp Neurol 248:190-222.

van den Berg AV (1992) Robustness of perception of heading from optic flow. Vision Res 32:1285-1296.

Warren WH, Hannon DJ (1988) Direction of self-motion is perceived from optical flow. Nature 336:162-163.

Warren WH, Hannon DJ (1990) Eye movements and optical flow. J Opt Soc Am A 7:160-169.

Warren WH, Morris MW, Kalish M (1988) Perception of translational heading from optic flow. J Exp Psychol (Hum Percept) 14:646-660.

Warren WH Jr, Blackwell AW, Kurtz KJ, Hatsopoulos NG, Kalish ML (1991) On the sufficiency of the velocity field for perception of heading. Biol Cybern 65:311-320.

Wurtz RH, Duffy CJ (1992) Neuronal correlates of optic flow stimulation. In: Sensing and controlling motion: vestibular and sensorimotor function (Cohen B, Tomko DL, Guedry F, eds), pp 205-219. New York: New York Academy of Science.

Wurtz RH, Yamasaki DS, Duffy CJ, Roy J-P (1990) Functional specialization for visual motion processing in primate cerebral cortex Cold Spring Harbor Symp Quant Biol 55:717-727.

Wurtz RH, Duffy CJ, Roy J-P (1993) Motion processing for guiding self-motion. In: Brain mechanisms of perception and memory: from neuron to behavior (Ono T, Squire LR, Raichle ME, Perrett D, Fukuda M, eds), pp 141-165. New York: Oxford UP. 